\title{
LATTICES IN THE COHOMOLOGY OF $U(3)$ ARITHMETIC MANIFOLDS
}

\author{
DANIEL LE
}

\begin{abstract}
Under hypotheses required for the Taylor-Wiles method, we prove for forms of $U(3)$ which are compact at infinity that the lattice structure on upper alcove algebraic vectors or on principal series types given by the $\lambda$-isotypic part of completed cohomology is a local invariant of the Galois representation attached to $\lambda$ when this Galois representation is residually irreducible locally at places dividing $p$. As a crucial input, we establish corresponding mod $p$ multiplicity one results. Our main innovation is the combination of integral Hecke theory and the Taylor-Wiles method.
\end{abstract}

\section{INTRODUCTION}

1.1. Background and the main result. Our goal is to prove a $p$-adic local-global compatibility result. Let $K / \mathbf{Q}_{p}$ be a finite extension and $\rho: G_{K} \rightarrow \mathrm{GL}_{n}\left(\overline{\mathbf{Q}}_{p}\right)$ a regular de Rham Galois representation. Let $\pi$ be the smooth $\mathrm{GL}_{n}(K)$-representation corresponding to the Weil-Deligne representation $\mathrm{WD}(\rho)$ via the local Langlands correspondence, and let $V$ be the algebraic $\mathrm{GL}_{n}(K)$-representation corresponding to the Hodge-Tate weights of $\rho$. If $n=2$ and $K=\mathbf{Q}_{p}$, the $p$-adic Langlands correspondence for $\mathrm{GL}_{2}\left(\mathbf{Q}_{p}\right)$, initiated in Bre03, and established in Col10, Paš13, CDP14, attaches to $\bar{\rho}$ a $p$-adic Banach space completion of $\pi \otimes V$. Moreover, the correspondence satisfies p-adic local-global compatibility by [Eme11, that is, the above completion coincides with the completion found in the $r$-part of completed cohomology of modular curves if $r: G_{\mathbf{Q}} \rightarrow \mathrm{GL}_{2}\left(\overline{\mathbf{Q}}_{p}\right)$ is a modular Galois representation whose restriction $\left.r\right|_{D_{p}}$ is isomorphic to $\rho$.

If $n>2$ or $K \neq \mathbf{Q}_{p}$, there is increasing evidence, though no definitive conjecture, for an analogous correspondence for $\mathrm{GL}_{n}(K)$ (see Bre10). However, if $\rho$ is the local restriction $\left.r\right|_{D_{v}}$ of a modular Galois representation $r: G_{F} \rightarrow \mathrm{GL}_{n}\left(\overline{\mathbf{Q}}_{p}\right)$ of a CM field $F$, then the completed cohomology of $U(n)$ arithmetic manifolds gives a natural integral structure, and thus a norm, on $\pi \otimes V$. Though this norm is of global origin, it is natural to hope for $p$-adic local-global compatibility results relating this norm to $\rho$, and thus informing our understanding of the hypothetical correspondence. In recent years, significant progress on $p$-adic local-global compatibility has been made in the case when $n=2$ and $K / \mathbf{Q}_{p}$ is an unramified extension, most notably the proof in EGS15, of Bre14, Conjecture 1.2]. If $\mathcal{O}_{K}$ denotes the ring of integers of $K$, then one can study $\mathrm{GL}_{n}(K)$-representations by studying certain $\mathrm{GL}_{n}\left(\mathcal{O}_{K}\right)$-subrepresentations called $\mathrm{GL}_{n}\left(\mathcal{O}_{K}\right)$-types. This is a powerful tool in the smooth representation theory of $p$-adic groups (see Bus95]), and it is natural to import it to the study of $p$-adic representations. EGS15] proves a $p$-adic local-global compatibility result for the integral structure on generic tame $\mathrm{GL}_{n}\left(\mathcal{O}_{K}\right)$-types. We 
prove analogous results for generic tame principal series $\mathrm{GL}_{3}\left(\mathbf{Z}_{p}\right)$-types in supersingular cases, to our knowledge, the first local-global compatibility results in the $p$-adic Langlands program for a group of semisimple rank greater than one.

We now explain our generalization in more detail. We now take $n$ to be 3 . Let $F$ be a $\mathrm{CM}$ field with totally real subfield $F^{+}$and assume that $F / F^{+}$is unramified at all finite places. Suppose that $p \neq 2$ splits completely in $F$. Let $E$ be a finite extension of $\mathbf{Q}_{p}$ with ring of integers $\mathcal{O}_{E}$, uniformizer $\varpi_{E}$, and residue field $k_{E}$ such that $r$ is defined over $E$. Suppose for simplicity that $r$ is unramified outside of $p$ (see Theorems 6.1.3 and 6.2.6 for weaker hypotheses). Let $\lambda$ be the Hecke eigensystem corresponding to $r$. Consider an integral model $\mathbf{U}_{/ \mathcal{O}_{F+}}$, which is reductive at all split places, for a form of $U(3)$ over $F^{+}$that splits over $F$, is quasisplit at all finite places, and is compact at infinity. Let $\widehat{H}^{0}$ be the completed cohomology with $\mathcal{O}_{E^{-}}$-coefficients of the associated arithmetic manifold (with tame level as described in $\$ 4.1 .3$ and assume that $\widehat{H}^{0}[\lambda] \neq 0$. For each place $v \mid p$ of $F^{+}$, choose a place $\widetilde{v} \mid v$ of $F$. Let $V_{\widetilde{v}}$ be the algebraic representation corresponding to the Hodge-Tate weights of $\left.r\right|_{D_{\tilde{v}}}$ and $\tau_{\widetilde{v}} \subset \pi_{\widetilde{v}}$ be the $\mathrm{GL}_{3}\left(\mathcal{O}_{F_{\tilde{v}}}\right)$-type and $\mathrm{GL}_{3}\left(F_{\widetilde{v}}\right)$-representation, respectively, corresponding to $\mathrm{WD}\left(\left.r\right|_{D_{\widetilde{v}}}\right)$ by the (inertial) local Langlands correspondence as in BC09, Proposition 6.5.3]. Since

$$
\operatorname{Hom}\left(\otimes_{v \mid p} \pi_{\widetilde{v}} \otimes V_{\widetilde{v}}, \widehat{H}^{0}[\lambda] \otimes_{\mathcal{O}_{E}} E\right) \cong \operatorname{Hom}\left(\otimes_{v \mid p} \tau_{\widetilde{v}} \otimes V_{\widetilde{v}}, \widehat{H}^{0}[\lambda] \otimes_{\mathcal{O}_{E}} E\right)
$$

(as $\prod_{v \mid p} \mathbf{U}\left(F_{v}^{+}\right)$and $\prod_{v \mid p} \mathbf{U}\left(\mathcal{O}_{F_{v}^{+}}\right)$-representations, respectively, under the natural isomorphism $\left.\iota_{\widetilde{v}}: \mathbf{U}\left(F_{v}^{+}\right) \stackrel{\sim}{\rightarrow} \mathrm{GL}_{3}\left(F_{\widetilde{v}}\right)\right)$ is one-dimensional over $E$ by Lab11, Theorems 5.4 and 5.9], the intersection $\left(\otimes_{v \mid p} \tau_{\widetilde{v}} \otimes V_{\widetilde{v}} \otimes_{\mathbf{Q}_{p}} E\right) \cap \widehat{H}^{0}[\lambda]$ gives a homothety class of lattices in $\otimes_{v \mid p} \tau_{\widetilde{v}} \otimes V_{\widetilde{v}} \otimes_{\mathbf{Q}_{p}} E$. In this paper we will study the following two situations: either $\left.r\right|_{D_{\widetilde{v}}}$ is crystalline with upper alcove Hodge-Tate weights, in which case $\tau_{\widetilde{v}}$ is trivial and $V_{\widetilde{v}}$ is what we will call an upper alcove algebraic representation (see \$1.4); or else $\left.r\right|_{D_{\tilde{v}}}$ is potentially crystalline of principal series Galois type, in which case $\tau_{\widetilde{v}}$ is a principal series type and $V_{\widetilde{v}}$ is trivial. The following is our main result.

Theorem 1.1.1. Suppose that for all places $v \mid p$ of $F^{+},\left.r\right|_{D_{\tilde{v}}}$ is crystalline of HodgeTate weights $\left(c_{\widetilde{v}}+p+1, b_{\widetilde{v}}+1, a_{\widetilde{v}}-p+1\right)$ or potentially crystalline at all places $v \mid p$ of Hodge-Tate weights $(0,1,2)$ and type $\eta^{-a_{\tilde{v}}} \oplus \eta^{-b_{\tilde{v}}} \oplus \eta^{-c_{\tilde{v}}}$. Suppose that $\bar{r}$ satisfies the Taylor-Wiles conditions, $\left.\bar{r}\right|_{G_{F_{\widetilde{v}}}}$ is irreducible for places $v \mid p$, and that $a_{\widetilde{v}}-b_{\widetilde{v}}>6, b_{\widetilde{v}}-c_{\widetilde{v}}>6$, and $a_{\widetilde{v}}-c_{\widetilde{v}}<p-5$. Then the homothety class of the lattice $\left(\otimes_{v \mid p} \tau_{\widetilde{v}} \otimes V_{\widetilde{v}} \otimes \mathbf{Q}_{p} E\right) \cap \widehat{H}^{0}[\lambda]$ in $\tau_{\widetilde{v}} \otimes V_{\widetilde{v}} \otimes \mathbf{Q}_{p} E$ can be described in terms of the crystalline Frobenius eigenvalues of $\left.r\right|_{D_{\tilde{v}}}$ (see Theorems 6.1.3 and 6.2.6 for the precise description).

Note that in the crystalline case with lower alcove Hodge-Tate weights, there is only one lattice up to homothety, and so the analogous result is trivial. In the $\mathrm{GL}_{2}\left(\mathbf{Q}_{p}\right)$ crystalline case, an analogous relationship between lattices in algebraic vectors and Hecke eigenvalues is used in BG09, BG13, BG15, BGR15, to compute the reduction of two-dimensional crystalline representations. See Corollary 6.1.5 for a partial result in this direction for $\mathrm{GL}_{3}\left(\mathbf{Q}_{p}\right)$. In the unramified $\mathrm{GL}_{2}$ tame principal series case, [BP12, Bre14] use analogous results to study the representations occuring in the $\bmod p$ cohomology of Shimura curves. It is natural to hope for a similar theory for the $U(3)$ arithmetic manifolds that we study. 
1.2. Methods and an overview of the paper. The first step, which is the subject of $\S_{2}$, is to classify lattices in $\tau_{\widetilde{v}} \otimes V_{\widetilde{v}}$. For the relevant algebraic representations, this follows from [Jan03, Part II]. For generic tame principal series types, there are partial classifications of lattices in the literature, e.g. in [CL76, §8] and [Jan84, using classical intertwiners for principal series. To get a full classification, it turns out to be sufficient to compute the submodule structure of the reduction of natural lattices in principal series types, which we compute using results of Her11a. The next step, which is the subject of $\$ 3$, is to isolate certain integral Hecke operators and show how they determine invariants of lattices. The computation of these integral Hecke operators can be seen as an extension of some results of Her11a. Finally, we combine our representation theoretic results with recent modifications of the Taylor-Wiles method (TW95]). Kisin's local-global modification ( Kis09]) provides a natural setting to compare the local and global Langlands correspondences integrally and in families. More specifically, we prove Theorem 1.1.1 by studying a patching functor applied to maps between lattices. The modification in $\left[\mathrm{CEG}^{+} 16\right]$ allows us to describe these maps in terms of Hecke operators. We describe the necessary results of $\left[\mathrm{CEG}^{+} 16\right.$ in $\$$. In $\$ 5$, we take the geometric perspective of [EG14] and EGS15] and prove Theorem 1.1.1 in families where one can use that patched modules are Cohen-Macaulay. The Cohen-Macaulay property is used in two essential ways. First, we use our representation theoretic results to augment (see Theorems 5.3.2 and 5.3.5 the argument of Dia97, Fuj06 that uses the Auslander-Buchsbaum formula to prove $\bmod p$ multiplicity one results. Second, we use that a generically vanishing submodule of a Cohen-Macaulay module is in fact zero to show that a single Hecke operator determines lattices in the crystalline case. Finally, in $\$ 6$, we use classical local-global compatibility to compute Hecke eigenvalues, and thus lattices in cohomology, from crystalline Frobenius eigenvalues of $\left.r\right|_{D_{\tilde{v}}}$.

1.3. Acknowledgments. This work grew out of my thesis. I am deeply indebted to my adviser Matthew Emerton for guidance, support, and numerous conversations about patching and the $p$-adic Langlands program. I thank Florian Herzig for correspondence regarding Proposition 2.2.2. I thank Christophe Breuil for sharing a letter about lattice conjectures for $\mathrm{GL}_{3}\left(\mathbf{Q}_{p}\right)$ which in addition to Bre14 inspired this paper. This paper also owes a debt to ideas (some unpublished and some in EGH13, EGS15, $\mathrm{CEG}^{+} 16$ ) of Ana Caraiani, Matthew Emerton, Toby Gee, David Geraghty, Florian Herzig, Vytautas Paskunas, David Savitt, and Sug Woo Shin, and it is a pleasure to acknowledge this. We thank Matthew Emerton, Toby Gee, Florian Herzig, and Bao V. Le Hung for comments on previous drafts of this paper. Finally, we heartily thank the referee for several comments which improved the exposition and accuracy of the paper.

1.4. Notation. For a field $k, G_{k}$ denotes the absolute Galois group of $k$. We denote the cyclotomic character by $\epsilon$. Hodge-Tate weights are normalized so that $\epsilon$ has weight -1 . We denote by $F^{+} \subset F$ the maximal totally real subfield of a CM field. The letter $v$ is used to denote places of $F^{+}$while the letter $w$ is used to denote places of $F$. Further, $\widetilde{v}$ denotes a place of $F$ dividing a place $v$ of $F^{+}$. We use $E$ to denote a finite extension of $\mathbf{Q}_{p}$ with ring of integers $\mathcal{O}_{E}$, uniformizer $\varpi_{E}$, and residue field $k_{E}$. 
Compact induction is denoted $\operatorname{ind}_{H}^{G}$, while usual induction is denoted $\operatorname{Ind}_{H}^{G}$. The symbols $(\cdot)^{\vee}$ and $(\cdot)^{d}$ are used to denote the Pontriagin dual and Schikhof dual, respectively (see $\left[\mathrm{CEG}^{+} 16, \S 1.8\right]$ ). The symbol $(\cdot)^{*}$ is used to denote the contragredient of a representation.

Let $\mathbf{B}$ and $\mathbf{T} \subset \mathrm{GL}_{n / \mathbf{Z}}$ be the algebraic subgroups of upper triangular and diagonal matrices, respectively. As usual, the group of characters (resp. cocharacters) of $\mathbf{T}$ are denoted by $X^{*}(\mathbf{T})$ (resp. $X_{*}(\mathbf{T})$ ). These groups are identified with $\mathbf{Z}^{n}$ in the usual way. Further subscripts with + (resp. -) denote dominant (resp. antidominant) characters and cocharacters. For a dominant character $\nu \in X^{*}(\mathbf{T})_{+}$, we say that $\nu$ is $p$-restricted if $0 \leq\left\langle\nu, \alpha^{\vee}\right\rangle<p$ for all simple positive $\operatorname{roots} \alpha$. If $\nu \in X^{*}(\mathbf{T})_{+}$is a dominant and $p$-restricted character, let $W(\nu)$ be the $\mathbf{Z}_{p}$-points of the algebraic $\mathrm{GL}_{n}$-representation $\operatorname{Ind}_{\mathbf{B}}^{\mathrm{GL}_{n}} w_{0} \nu$, where $w_{0}$ denotes the longest element of $S_{n}$. Let $\bar{W}(\nu)$ be the reduction of $W(\nu)$ modulo $p$, and let $F(\nu)$ be the (irreducible) socle of $\bar{W}(\nu)$. These characteristic $p$ representations factor through $\mathrm{GL}_{n}\left(\mathbf{F}_{p}\right)$. Every (absolutely) irreducible representation of $\mathrm{GL}_{n}\left(\mathbf{F}_{p}\right)$ is isomorphic to $F(\nu)$ for some $\nu$, and the representations $F(\mu)$ and $F(\nu)$ are isomorphic if and only if $\mu \equiv \nu(\bmod p-1)$ (see [Her09, Theorem 3.10]). The representations $F(\nu)$ are called (Serre) weights (of $\mathrm{GL}_{n}\left(\mathbf{F}_{p}\right)$ ).

Usually, we will take $n$ above to be 3 . We label the elements of $S_{3}$ as $e=()$, $s_{1}=(12), s_{2}=(23), r_{1}=(123), r_{2}=(132)$, and $w_{0}=(13)$. The ordered pair $(i, j)$ often denotes some permutation of the ordered pair $(1,2)$. Let $(x, y, z) \in X^{*}(\mathbf{T})_{+}$. The character $(x, y, z)$ is in the lower alcove (resp. upper alcove) if $x-z<p-2$ (resp. $x-y, y-z<p-1$ and $p-2<x-z$ ) (see the paragraph following Her06, Corollary 4.8]). We say that $F(\nu)$ is a lower (resp. upper) alcove Serre weight if $\nu$ is a lower (resp. upper) alcove character.

The triple $(a, b, c)$ with $a>b>c$ will be used to denote the highest weight of an algebraic representation of $\mathrm{GL}_{3}\left(\mathbf{F}_{p}\right)$. We will assume throughout (with the exception of $\$ 2.2$ that $a-c<p$ and usually that $a-c<p-1$. In $\$ 6$, we assume that $a-b>6, b-c>6$, and $a-c<p-5$ (the strong genericity hypothesis of [EGH13]) to apply [EGH13, Theorem 7.5.5].

By [And87, Theorem 2.8 and $\S 4$ ], we have the following results. Note that And87. describes $\mathrm{SL}_{3}\left(\mathbf{F}_{p}\right)$-representations, but the proofs carry over verbatim to the $\mathrm{GL}_{3}\left(\mathbf{F}_{p}\right)$-setting. Alternatively, the results in the $\mathrm{GL}_{3}\left(\mathbf{F}_{p}\right)$-setting can be easily deduced from the $\mathrm{SL}_{3}\left(\mathbf{F}_{p}\right)$-setting via the inflation-restriction exact sequence. If $\sigma$ and $\sigma^{\prime}$ are weights, then

$$
\operatorname{dim}_{\mathbf{F}_{p}} \operatorname{Ext}_{\mathrm{GL}_{3}\left(\mathbf{F}_{p}\right)}^{1}\left(\sigma^{\prime}, \sigma\right)=\operatorname{dim}_{\mathbf{F}_{p}} \operatorname{Ext}_{\mathrm{GL}_{3}\left(\mathbf{F}_{p}\right)}^{1}\left(\sigma, \sigma^{\prime}\right) \leq 1 .
$$

If $F(x, y, z)$ is a weight, let $\mathcal{E}(x, y, z)$ be the set of triples $(a, b, c)$ such that

$$
\operatorname{Ext}_{\mathrm{GL}_{3}\left(\mathbf{F}_{p}\right)}^{1}(F(x, y, z), F(a, b, c)) \neq 0 .
$$

If $(x, y, z)$ is a lower alcove character, then

$$
\begin{aligned}
\mathcal{E}(x, y, z):=\{ & (y+p-1, x, z),(x, z, y-p+1),(z+p-2, y, x-p+2), \\
& (x+1, z-1, y-p+1),(y+p-1, x+1, z-1),(x, z-1, y-p+2), \\
& (y+p-2, x+1, z)\} .
\end{aligned}
$$

Moreover, $\operatorname{Ext}_{\mathrm{GL}_{3}\left(\mathbf{F}_{p}\right)}^{1}\left(\sigma^{\prime}, \sigma\right)=0$ if both $\sigma$ and $\sigma^{\prime}$ are upper alcove weights. 


\section{LATTICES IN LOCALLY ALGEBRAIC REPRESENTATIONS}

Let $\tau$ be either an upper alcove algebraic representation or a sufficiently generic principal series type over E. Then $\tau$ is residually multiplicity free in the sense that the semisimplification $\bar{\tau}^{\mathrm{ss}}$ of the reduction of any lattice in $\tau$ is multiplicity free. By EGS15, Lemma 4.1.1], this condition implies that for each Jordan-Hölder factor $\sigma$ of $\bar{\tau}^{\text {ss }}$, there is up to homothety a unique lattice $\tau^{\sigma}$ in $\tau$ with cosocle isomorphic to $\sigma$. In this section we make a detailed study of the lattices $\tau^{\sigma}$ (and the relations between them for varying $\sigma$ ) for upper alcove algebraic representations (2.1) and principal series types $(2.2$. In 2.3 , we use Morita theory to explain how this information leads to a classification of lattices in these representations.

2.1. Lattices in algebraic vectors. In this section, we define two natural integral structures on upper alcove algebraic vectors. We follow the notation of [Her09, §3]. Let $K$ be $\mathrm{GL}_{3}\left(\mathbf{Z}_{p}\right)$ and $K_{1}$ be the kernel of the reduction map $\mathrm{GL}_{3}\left(\mathbf{Z}_{p}\right) \rightarrow \mathrm{GL}_{3}\left(\mathbf{F}_{p}\right)$.

Fix $a>b>c$ integers such that $a-c<p$. Note that $(a-1, b, c+1)$ is a lower alcove character. Let $\nu$ be the ( $p$-restricted) upper alcove character $(c+p-1, b, a-$ $p+1)$. Throughout this paper, we will denote $W(\nu)$ and $W\left(-w_{0} \nu\right)^{*}$ by $W$ and $W^{0}$, respectively, where $*^{*}$ denotes the contragredient representation. By Serre duality for $\mathrm{GL}_{3} / \mathbf{B}$, there is an isomorphism $W^{0} \cong \mathrm{R}^{3} \operatorname{Ind}_{\mathbf{B}}^{\mathrm{GL}_{3}}(\nu-2 \rho)\left(\mathbf{Z}_{p}\right)$ where $2 \rho$ is the sum of the positive roots of $\mathrm{GL}_{3}$ (see [Jan03, Chapter II.4(8)]).

Let $V=W\left[p^{-1}\right]=\operatorname{Ind}_{\mathbf{B}}^{\mathrm{GL}_{3}} w_{0} \nu\left(\mathbf{Q}_{p}\right)$. By the Borel-Weil-Bott theorem, $V \cong$ $\mathrm{R}^{3} \operatorname{Ind}_{\mathbf{B}}^{\mathrm{GL}_{3}}(\nu-2 \rho)\left(\mathbf{Q}_{p}\right) \cong W^{0}\left[p^{-1}\right]$ (see [Jan03, II.5.3]), and so $W^{0}$ is (isomorphic to) a lattice in $V$. The next proposition shows that the lattices $W$ and $W^{0}$ are the two lattices up homothety with irreducible cosocle. Let $\bar{W}$ and $\bar{W}^{0}$ be the reductions modulo $p$ of $W$ and $W^{0}$, respectively. Note that $K_{1}$ acts trivially on $\bar{W}$ and $\bar{W}^{0}$.

Proposition 2.1.1. There are nonsplit exact sequences

$$
0 \rightarrow F(c+p-1, b, a-p+1) \rightarrow \bar{W} \rightarrow F(a-1, b, c+1) \rightarrow 0
$$

and

$$
0 \rightarrow F(a-1, b, c+1) \rightarrow \bar{W}^{0} \rightarrow F(c+p-1, b, a-p+1) \rightarrow 0
$$

of $K$-representations.

Proof. The first exact sequence follows from [Her06, Proposition 4.9]. The nonsplitness follows from [Her06, Theorem 4.1]. The second nonsplit exact sequence follows similarly after applying contragredients, where we use that the contragredient of $F(x, y, z)$ is $F(-z,-y,-x)$.

Fix an injection $i: W^{0} \hookrightarrow W$ that is nonzero modulo $p$ (see [Jan03, II.8.13] for particular choices) and let $i^{\vee}: W \hookrightarrow W^{0}$ be the unique map that is nonzero modulo $p$ such that $i \circ i^{\vee}$ is a power of $p$.

Proposition 2.1.2. The composition $i \circ i^{\vee}$ is $p$.

Proof. This follows from [Jan03, II.8.15 (3)(4)]. We take $G$ to be $\mathrm{GL}_{3}$ so that $H^{i}$ in loc. cit. is $\mathrm{R}^{i} \operatorname{Ind}_{\mathbf{B}}^{\mathrm{GL}_{3}}$. We take $\mu$ to be $w_{0} \nu$ so that $w_{0} \cdot \mu=\nu-2 \rho$. Let $\alpha_{1}$ and $\alpha_{2}$ be $(1,-1,0)$ and $(0,1,-1)$, respectively. Then $s_{\alpha_{i}}$ in loc. cit. is $s_{i}$. Since $\widetilde{T}_{\alpha}\left(s_{\alpha} \cdot \mu\right) \circ$ $\widetilde{T}_{\alpha}(\mu)$ is multiplication by $\left(\left\langle\mu, \alpha^{\vee}\right\rangle\right)$ ! for all $\mu$, we see that $\widetilde{T}_{\alpha_{1}}(\mu)$ and $\widetilde{T}_{\alpha_{1}}\left(s_{2} s_{1} \cdot \mu\right)$ are isomorphisms while $\widetilde{T}_{\alpha_{2}}\left(s_{2} s_{1} \cdot \mu\right) \circ \widetilde{T}_{\alpha_{2}}\left(s_{1} \cdot \mu\right)$ is, up to a unit, multiplication by $p$. 
As $\widetilde{T}_{\alpha}(\mu)$ is nonzero for all $\mu$, we can take $i=\widetilde{T}_{\alpha_{1}}\left(s_{1} \cdot \mu\right) \circ \widetilde{T}_{\alpha_{2}}\left(s_{2} s_{1} \cdot \mu\right) \circ \widetilde{T}_{\alpha_{1}}\left(w_{0} \cdot \mu\right)$ and $i^{\vee}$ to be a unit multiple of $\widetilde{T}_{\alpha_{1}}\left(s_{2} s_{1} \cdot \mu\right) \circ \widetilde{T}_{\alpha_{2}}\left(s_{1} \cdot \mu\right) \circ \widetilde{T}_{\alpha_{1}}(\mu)$. This gives the desired result.

2.2. Lattices in principal series types. In this section, we classify lattices in generic principal series types which are residually multiplicity free.

2.2.1. Principal series types in characteristic $p$. We describe the submodule structure of principal series types in characteristic $p$. We begin with the submodule struc-

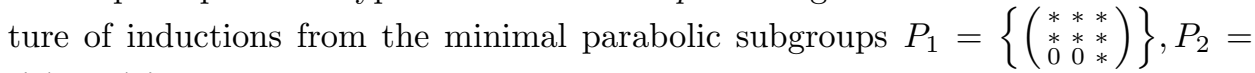
$\left\{\left(\begin{array}{lll}* & * & * \\ 0 & * & * \\ 0 & * & *\end{array}\right)\right\}$ of $\mathrm{GL}_{3}\left(\mathbf{F}_{p}\right)$, strengthening [EGH13, Lemma 6.1.1].

For $s \in S_{3}$, let $s(a, b, c)$ be a dominant $p$-restricted character congruent mod $p-1$ to the $s$-permutation of $(a, b, c)$ (e.g. if $(a, b, c)$ is $p$-restricted, then $r_{1}(a, b, c)=$ $(c+p-1, a, b))$.

Proposition 2.2.1. Let $a, b$, and $c$ be integers such that $0<a-b<p-1$ and $0<b-c<p-1$.

(1) The socle and cosocle of $\operatorname{Ind}_{P_{1}}^{\mathrm{GL}_{3}\left(\mathbf{F}_{p}\right)} F(a, b) \otimes F(c)$ are isomorphic to $F\left(r_{1}(a, b, c)\right)$ and $F(a, b, c)$, respectively. The socle and cosocle filtration have three nonzero graded pieces.

(2) The socle and cosocle of $\operatorname{Ind}_{P_{2}}^{\mathrm{GL}_{3}\left(\mathbf{F}_{p}\right)} F(a) \otimes F(b, c)$ are isomorphic to $F\left(r_{2}(a, b, c)\right)$ and $F(a, b, c)$, respectively. The socle and cosocle filtration have three nonzero graded pieces.

Proof. We prove only the first item as the proof of the second item is similar. For $i=1$ and 2 , let $N_{i} \subset P_{i}$ be the maximal normal $p$-subgroup, and $M_{i}=P_{i} / N_{i}$. We begin by calculating the cosocle of $\operatorname{Ind}_{P_{1}}^{\mathrm{GL}_{3}\left(\mathbf{F}_{p}\right)} F(a, b) \otimes F(c)$. By Frobenius reciprocity,

$\operatorname{Hom}_{\mathrm{GL}_{3}\left(\mathbf{F}_{p}\right)}\left(\operatorname{Ind}_{P_{1}}^{\mathrm{GL}_{3}\left(\mathbf{F}_{p}\right)} F(a, b) \otimes F(c), F(x, y, z)\right) \cong \operatorname{Hom}_{M_{1}}\left(F(a, b) \otimes F(c), F(x, y, z)^{N_{1}}\right)$.

As $F(x, y) \otimes F(z) \subset F(x, y, z)^{N_{1}}$, and the latter is irreducible by [Her11a, Lemma $2.5(\mathrm{i})]$, we have that $F(x, y, z)^{N_{1}} \cong F(x, y) \otimes F(z)$. Thus the Hom-space above is nonzero if and only if it is one-dimensional and $F(x, y, z) \cong F(a, b, c)$.

The socle computation is similar. By Frobenius reciprocity,

$\operatorname{Hom}_{\mathrm{GL}_{3}\left(\mathbf{F}_{p}\right)}\left(F(x, y, z), \operatorname{Ind}_{P_{1}}^{\mathrm{GL}_{3}\left(\mathbf{F}_{p}\right)} F(a, b) \otimes F(c)\right) \cong \operatorname{Hom}_{M_{1}}\left(F(x, y, z)_{N_{1}}, F(a, b) \otimes F(c)\right)$.

By [Her11a, Lemma 2.5(ii)], $F(x, y, z)_{N_{1}} \cong F(x, y, z)^{\bar{N}_{1}}$. Since $r_{1} \bar{N}_{1} r_{1}^{-1}=N_{2}$ and $F(x, y, z)^{N_{2}} \cong F(x) \otimes F(y, z)$, we see that $F(x, y, z)^{\bar{N}_{1}} \cong F(y, z) \otimes F(x)$. Arguing as in the previous paragraph, we see that $(a, b, c) \equiv(y, z, x) \bmod (p-1)$ and that $F(x, y, z)=F(c+p-1, a, b)$.

The remaining Jordan-Hölder factors of $\operatorname{Ind}_{P_{1}}^{\mathrm{GL}_{3}\left(\mathbf{F}_{p}\right)} F(a, b) \otimes F(c)$ can be determined by the analogue of [EGH13, Lemma 6.1.1]. The crucial observation is that the remaining Jordan-Hölder factors do not admit extensions between them since they all lie in the same alcove. Thus the socle and cosocle filtrations have exactly three nonzero graded pieces.

Let $I \subset K$ (resp. $I_{1} \subset K$ ) be the inverse image of $\mathbf{B}\left(\mathbf{F}_{p}\right) \subset \mathrm{GL}_{3}\left(\mathbf{F}_{p}\right)$ (resp. maximal $p$-subgroup of $\mathbf{B}\left(\mathbf{F}_{p}\right)$ ) under the reduction map $K \rightarrow \mathrm{GL}_{3}\left(\mathbf{F}_{p}\right)$. Let $\bar{\eta}: \mathbf{F}_{p}^{\times} \rightarrow \mathbf{F}_{p}^{\times}$be the identity character. For integers $a, b$, and $c$, we consider the 
character $\bar{\chi}=\bar{\eta}^{a} \otimes \bar{\eta}^{b} \otimes \bar{\eta}^{c}$ of $\left(\mathbf{F}_{p}^{\times}\right)^{3}$, which we view as a character of $I$ by inflation via the usual map $I \rightarrow I / I_{1} \stackrel{\sim}{\rightarrow}\left(\mathbf{F}_{p}^{\times}\right)^{3}$. Let $\bar{\tau}$ be the $K$-representation $\operatorname{ind}_{I}^{K} \bar{\chi}$ over $\mathbf{F}_{p}$. We suppose that $a, b$, and $c$ are integers such that $0<a-b<p-1$, $0<b-c<p-1$, and $a, b$, and $c$ are distinct modulo $p-1$. The following proposition describes the submodule structure of $\bar{\tau}$. Its proof was explained to us by Florian Herzig.

Proposition 2.2.2. If $a-c<p-1$ or $a-c>p-1$, then the socle and cosocle filtrations of $\bar{\tau}$ agree and have associated graded pieces

$$
\begin{gathered}
F(a, b, c) \\
F(a, c, b-p+1) \oplus F(b+p-1, a, c) \\
F(c+p-1, a, b) \oplus F(b-1, c, a-p+2) \oplus F(a-1, b, c+1) \\
\oplus F(c+p-2, a, b+1) \oplus F(b, c, a-p+1) \\
F(c+p-1, b, a-p+1),
\end{gathered}
$$

or

$$
\begin{gathered}
F(a, b, c) \\
F(a, c+p-1, b) \oplus F(b+p-2, a, c+p) \oplus F(c+p-2, b, a-p+2) \\
\oplus F(a-p, c, b-p+2) \oplus F(b, a-p+1, c) \\
F(c+2 p-2, a, b) \oplus F(b, c, a-2 p+2) \\
F(c+p-1, b, a-p+1),
\end{gathered}
$$

respectively, where any number of bottom rows are the graded pieces of a submodule. Furthermore, all nontrivial extensions that can occur do occur (see \$1.4).

Proof. As $a, b$, and $c$ are distinct $\bmod p-1$, the Jordan-Hölder factors of $\tau^{e}$ are distinct by [Her06, Proposition 3.1, Theorems 4.1 and 5.1] and Proposition 2.1.1. Exactly as in the proof of Proposition 2.2.1, one can use Frobenius reciprocity and Her11a, Lemma 2.5], to show that the socle and cosocle of $\bar{\tau}$ are $F(c+p-1, b, a-$ $p+1)$ and $F(a, b, c)$, respectively. Hence, $F(c+p-1, b, a-p+1)$ and $F(a, b, c)$ are pieces of the associated graded for both the socle and cosocle filtrations.

We now assume that $a-c<p-1$. The other case can be treated similarly, or by using duality. By [EGH13, Lemma 6.1.1], there is a natural inclusion $\operatorname{Ind}_{P_{1}}^{\mathrm{GL}_{3}\left(\mathbf{F}_{p}\right)} F(b+p-1, a) \otimes F(c) \subset \bar{\tau}$. From Proposition 2.2.1, we see that in the socle filtration, $F(b-1, c, a-p+2), F(a-1, b, c+1), F(c+p-2, a, b+1)$, and $F(b, c, a-p+1)$ are in the layer above the socle $F(c+p-1, b, a-p+1)$, and 
$F(b+p-1, a, c)$ is in the next layer up as taking socles respects inclusions. Furthermore, all possible nontrivial extensions between these weights occur. The same argument applies to $\operatorname{Ind}_{P_{2}}^{\mathrm{GL}_{3}\left(\mathbf{F}_{p}\right)} F(a) \otimes F(c, b-p+1)$. The only remaining weight is the cosocle $F(a, b, c)$, which must be in the final layer of the socle filtration. Furthermore, since the cosocle is isomorphic to $F(a, b, c)$, this weight must extend $F(b+p-1, a, c)$ and $F(a, c, b-p+1)$. Finally, we observe that every irreducible factor of $\bar{\tau}$, aside from $F(a, b, c)$, is extended nontrivially by at least one irreducible factor in row of the socle filltration immediately above it, which implies that the cosocle filtration coincides with the socle filtration.

2.2.2. Integral structure on principal series types. We begin by defining natural integral structures on principal series. Let $\eta: \mathbf{F}_{p}^{\times} \rightarrow \mathbf{Z}_{p}^{\times}$be the Teichmuller character. For integers $a, b$, and $c$, we consider the character $\chi=\eta^{a} \otimes \eta^{b} \otimes \eta^{c}$ of $\left(\mathbf{F}_{p}^{\times}\right)^{3}$, which we view as a character of $I$ by inflation via the map $I \rightarrow I / I_{1} \stackrel{\sim}{\rightarrow}\left(\mathbf{F}_{p}^{\times}\right)^{3}$. Let $\tau^{e}$ be the $K$-representation ind $I_{I}^{K} \chi$ over $\mathbf{Z}_{p}$, and let $\tau=\tau^{e} \otimes \mathbf{z}_{p} \mathbf{Q}_{p}$. As in 2.2 .1 suppose that $a, b$, and $c$ are distinct modulo $p-1$, so that in particular $\tau$ is absolutely irreducible. By reordering $a, b$, and $c$ and adding multiples of $p-1$, let us now assume without loss of generality that $a>b>c$ and $a-c<p-1$. Let $\chi^{s}$ be the character with factors permuted by $s \in S_{3}$. Let $e$ be the identity, $w_{0}=(13), r_{1}=(123)$, $r_{2}=(132), s_{1}=(12)$, and $s_{2}=(23)$. Then for example $\chi^{r_{1}}=\eta^{c} \otimes \eta^{a} \otimes \eta^{b}$ and $\chi^{r_{2}}=\eta^{b} \otimes \eta^{c} \otimes \eta^{a}$.

For each $s \in S_{3}$, consider the $K$-representation $\tau^{s}=\operatorname{ind}_{I}^{K} \chi^{s}$ over $\mathbf{Z}_{p}$. The representations $\tau^{s}$ are lattices in the principal series types $\tau^{s} \otimes \mathbf{z}_{p} \mathbf{Q}_{p} \cong \tau$. Then by Proposition 2.2.2 and EGS15, Lemma 4.1.1], for each $s \in S_{3}$, the lattice $\tau^{s}$ is the unique lattice up to homothety with cosocle $F(s(a, b, c))$. Having described the reductions of lattices in $\tau$ with cosocle $F(s(a, b, c))$ in Proposition 2.2.2, we now describe the submodule structure of $\bar{\tau}^{1}, \bar{\tau}^{2}$, and $\bar{\tau}^{3}$ where $\tau^{1}, \tau^{2}$, and $\tau^{3} \subset \tau$ are the unique lattices up to homothety with cosocle $F(b-1, c, a-p+2), F(a-1, b, c+1)$, and $F(c+p-2, a, b+1)$, respectively.

Proposition 2.2.3. For $i=1,2$, or 3 , the cosocle filtration of $\bar{\tau}^{i}$ has three associated graded pieces. The quotient is an irreducible lower alcove weight, the next layer is the direct sum of the upper alcove weights in $\mathrm{JH}\left(\bar{\tau}^{i}\right)$, and the final layer is the direct sum of the remaining lower alcove weights in $\mathrm{JH}\left(\bar{\tau}^{i}\right)$. For example, for $\bar{\tau}^{1}$, the associated graded pieces are

$$
\begin{gathered}
F(b-1, c, a-p+2) \\
F(b+p-1, a, c) \oplus F(a, c, b-p+1) \oplus F(c+p-1, b, a-p+1) \\
F(a, b, c) \oplus F(b, c, a-p+1) \oplus F(c+p-1, a, b) \\
\oplus F(c+p-2, a, b+1) \oplus F(a-1, b, c+1) .
\end{gathered}
$$

Proof. We will prove the proposition for $\bar{\tau}^{1}$, the other cases being similar. The weight $F(b-1, c, a-p+2)$ is in the top layer for both filtrations by construction. Recall from the proof of [EGS15, Lemma 4.1.1], $\tau^{1} \subset \tau^{w_{0}}$ is the minimal submodule 
such that the quotient $\tau^{w_{0}} / \tau^{1}$ does not contain $F(b-1, c, a-p+2)$ as a JordanHölder factor. We claim that the image of $\bar{\tau}^{1}$ in $\bar{\tau}^{w_{0}}$ is the minimal submodule $M$ of $\bar{\tau}^{w_{0}}$ containing $F(b-1, c, a-p+2)$ as a Jordan-Hölder factor. It clearly must contain it. On the other hand, the quotient of $\tau^{w_{0}}$ by the preimage of $M$ does not contain $F(b-1, c, a-p+2)$ as a Jordan-Hölder factor, and so the preimage of $M$ must contain $\tau^{1}$ by minimality of $\tau^{1}$.

Proposition 2.2.2 shows that $F(a, c, b-p+1)$ and $F(b+p-1, a, c)$ are in the second layer of the cosocle filtration of $\mathrm{im} \bar{\tau}^{1}$. Since formation of the radical submodule commutes with quotients, $F(a, c, b-p+1)$ and $F(b+p-1, a, c)$ are in the second layer of the cosocle filtration of $\bar{\tau}^{1}$. Similarly, looking at the inclusion $\tau^{1} \subset \tau^{s_{1}}$, we see that $F(c+p-1, b, a-p+1)$ is also in the second layer of the cosocle filtration of $\bar{\tau}^{1}$. In fact, $F(a, c, b-p+1) \oplus F(b+p-1, a, c) \oplus F(c+p-1, b, a-p+1)$ is the second layer of the cosocle filtration since these weights are the only Jordan-Hölder factors of $\bar{\tau}^{1}$ that nontrivially extend $F(b-1, c, a-p+2)$ (see 1.4 . The other five weights are all in the third layer of the cosocle filtration since there are no nontrivial extensions between them (see 1.4 ).

For $s, s^{\prime} \in S_{3}$, the intertwiner $\iota_{s^{\prime} s}^{s}: \operatorname{ind}_{I}^{K} \chi^{s^{\prime} s} \hookrightarrow \operatorname{ind}_{I}^{K} \chi^{s}$ from the classical representation theory of $\mathrm{GL}_{3}\left(\mathbf{F}_{p}\right)$ is nonzero modulo $p$ and induces isomorphisms $\tau^{s^{\prime} s} \otimes \mathbf{z}_{p} \mathbf{Q}_{p} \stackrel{\sim}{\rightarrow} \tau^{s} \otimes \mathbf{z}_{p} \mathbf{Q}_{p}$, as we now recall.

Let $v^{s} \in \operatorname{ind}_{I}^{K} \chi^{s}$ be the function supported on $I$ defined by extending $\chi^{s}$ by 0 . By Frobenius reciprocity, the intertwiner $\iota_{s^{\prime} s}^{s}$ is determined by the image of $v^{s^{\prime} s}$. Identifying $S_{3}$ with the group of permutation matrices in $\mathrm{GL}_{3}$, the intertwiner is given by

$$
\iota_{s^{\prime} s}^{s}: v^{s^{\prime} s} \mapsto \sum_{g \in I_{1} /\left(s^{\prime} I_{1} s^{\prime-1} \cap I_{1}\right)} g s^{\prime} v^{s},
$$

which is easily checked to be nonzero modulo $p$. Let $\ell$ denote the length function on $S_{3}$.

Proposition 2.2.4. The composition $\iota_{s^{\prime} s}^{s^{\prime \prime} s^{\prime} s} \circ \iota_{s}^{s^{\prime} s}: \operatorname{ind}_{I}^{K} \chi^{s} \hookrightarrow \operatorname{ind}_{I}^{K} \chi^{s^{\prime \prime} s^{\prime} s}$ is $p^{\frac{1}{2}\left(\ell\left(s^{\prime \prime}\right)+\ell\left(s^{\prime}\right)-\ell\left(s^{\prime \prime} s^{\prime}\right)\right)} \iota_{s}^{s^{\prime \prime} s^{\prime} s}$.

Proof. A more general result can be obtained from [CL76, Propositions 3.6 and 3.10]. We provide a short summary. Let the ordered pair $(i, j)$ be a permutation of $(1,2)$. By induction, it suffices to show that $\iota_{s_{i} s}^{s_{j} s_{i} s} \circ \iota_{s}^{s_{i} s}=\iota_{s}^{s_{j} s_{i} s}$ and $\iota_{s_{i} s}^{s_{i} s_{i} s} \circ \iota_{s}^{s_{i} s}=p$.

Note that $\cup_{g \in I_{1} /\left(s^{\prime} I_{1} s^{\prime-1} \cap I_{1}\right)} g s^{\prime} I_{1}=I_{1} s^{\prime} I_{1}$. To prove that $\iota_{s_{i} s}^{s_{j} s_{i} s} \circ \iota_{s}^{s_{i} s}=\iota_{s}^{s_{j} s_{i} s}$, it suffices to show that the convolution of $\mathbf{1}_{I_{1} s_{j} I_{1}}$ and $\mathbf{1}_{I_{1} s_{i} I_{1}}$ is $\mathbf{1}_{I_{1} s_{j} s_{i} I_{1}}$. Since $I_{1} s_{j} s_{i} I_{1} \subset I_{1} s_{j} I_{1} \cdot I_{1} s_{i} I_{1}$, we have that $\mathbf{1}_{I_{1} s_{j} s_{i} I_{1}} \leq \mathbf{1}_{I_{1} s_{j} I_{1}} * \mathbf{1}_{I_{1} s_{i} I_{1}}$. As \# $I_{1} s_{j} s_{i} I_{1} / I_{1}=$ $\# I_{1} s_{i} I_{1} / I_{1} \cdot \# I_{1} s_{j} I_{1} / I_{1}, \mathbf{1}_{I_{1} s_{j} s_{i} I_{1}}$ and $\mathbf{1}_{I_{1} s_{j} I_{1}} * \mathbf{1}_{I_{1} s_{i} I_{1}}$ have the same integrals for any Haar measure. We conclude that they are equal. The identity $\iota_{s_{i} s}^{s_{i} s_{i} s} \circ \iota_{s}^{s_{i} s}=p$ reduces to a calculation for the $\mathrm{GL}_{2}$ minor coming from the $i$-th and $i+1$-th rows and columns. This identity then follows from the computation in the proof of Bre14, Lemme 2.2].

We now use Proposition 2.2.6 to complete the classification of maps between lattices with irreducible cosocle. For $i=1,2,3, s \in S_{3}$, let $\iota_{i}^{s}: \tau^{i} \rightarrow \tau^{s}$ be fixed inclusions of lattices that are nonzero modulo $p$. For $i$ and $j$ in the set $\{1,2,3\}$ with $i<j$, let $\iota_{i}^{j}: \tau^{i} \rightarrow \tau^{j}$ be fixed inclusions of lattices that are nonzero modulo p. These inclusions are unique up to unit scalar by [EGS15, Lemma 4.1.1]. Let 
$\iota_{s}^{i}: \tau^{s} \rightarrow \tau^{i}$ and $\iota_{j}^{i}: \tau^{j} \rightarrow \tau^{i}$ be the unique inclusions of lattices that are nonzero modulo $p$ such that $\iota_{s}^{i} \circ \iota_{i}^{s}$ and $\iota_{j}^{i} \circ \iota_{i}^{j}$ are powers of $p$.

Proposition 2.2.5. The compositions of inclusions of lattices are given as follows:

(1) $\iota_{j}^{i} \circ \iota_{i}^{j}=p^{2}$;

(2) if $s=s_{1}, s_{2}$, or $w_{0}$, then $\iota_{i}^{s} \circ \iota_{s}^{i}=p$; and

(3) if $s=e, r_{1}$, or $r_{2}$, then $\iota_{i}^{s} \circ \iota_{s}^{i}=p^{2}$.

Proof of (2). By relabelling $a, b$, and $c$, we can assume without loss of generality that $i=1$. We use the symbol $\sim$ to denote equality up to a unit. Let $s^{\prime}$ be one of $s_{1}, s_{2}$, or $w_{0}$ with $s^{\prime} \neq s$. By Proposition 2.2.3, the minimal submodule of $\bar{\tau}^{1}$ containing $F\left(s^{\prime}(a, b, c)\right)$ as a Jordan-Hölder factor does not contain $F(s(a, b, c))$ as a Jordan-Hölder factor. Thus, the image of the composition $\iota_{s^{\prime}}^{1} \circ \iota_{s}^{s^{\prime}}$ is zero modulo $p$, and hence a positive power of $p$ times $\iota_{s}^{i}$ up to a unit. Then again using Proposition 2.2.2. we have $p^{2}=\iota_{s^{\prime}}^{s} \circ \iota_{s}^{s^{\prime}} \sim \iota_{1}^{s} \circ \iota_{s^{\prime}}^{1} \circ \iota_{s}^{s^{\prime}}$ so that $p^{2}$ is a positive power of $p$ times $\iota_{1}^{s} \circ \iota_{s}^{1}$. We conclude that $\iota_{1}^{s} \circ \iota_{s}^{1}=p$.

To prove (1) and (3), we need the following result.

Proposition 2.2.6. For $i=1,2$, or 3 , the socle and cosocle filtrations of $\bar{\tau}^{i}$ agree and have three associated graded pieces (see Proposition 2.2.3). Moreover, all nontrivial extensions between pieces that can occur do occur (see \$1.4).

Proof. We first show the second statement. Clearly all possible nontrivial extensions between the top two rows occur. We now show that all possible nontrivial extensions between the bottom two rows occur. Since the composition $\iota_{1}^{s_{1}} \circ \iota_{s_{1}}^{1}=p$ by Proposition 2.2.5 (2), the image of $\iota_{1}^{s_{1}}: \bar{\tau}^{1} \rightarrow \bar{\tau}^{s_{1}}$ and the cokernel of $\iota_{s_{1}}^{1}: \bar{\tau}^{s_{1}} \rightarrow \bar{\tau}^{1}$ have the same Jordan-Hölder factors, namely $F(b-1, c, a-p+2)$, $F(a, c, b-p+1), F(c+p-1, b, a-p+1)$, and $F(c+p-1, a, b)$ by Proposition 2.2.2. Hence all possible nontrivial extensions of $F(b+p-1, a, c)$ occur in $\bar{\tau}^{1}$ since they occur in $\operatorname{im}\left(\bar{\tau}^{s_{1}} \rightarrow \bar{\tau}^{1}\right)$ by Proposition 2.2.2. All other nontrivial extensions are established analogously. The first statement follows from Proposition 2.2.3 and the second statement.

Proof of Proposition 2.2.5(1) and (3). We now turn to (1). We continue to use the symbol $\sim$ to denote equality up to a unit. By Propositions 2.2.2 and 2.2.6, we have $\iota_{s_{1}}^{w_{0}} \sim \iota_{i}^{w_{0}} \circ \iota_{s_{1}}^{i}, \iota_{w_{0}}^{s_{1}} \sim \iota_{j}^{s_{1}} \circ \iota_{w_{0}}^{j}, \iota_{j}^{i} \sim \iota_{s_{1}}^{i} \circ \iota_{j}^{s_{1}}$, and $\iota_{i}^{j} \sim \iota_{w_{0}}^{j} \circ \iota_{i}^{w_{0}}$. Hence $\iota_{i}^{w_{0}} \circ \iota_{j}^{i} \circ \iota_{i}^{j} \sim \iota_{i}^{w_{0}} \circ \iota_{s_{1}}^{i} \circ \iota_{j}^{s_{1}} \circ \iota_{w_{0}}^{j} \circ \iota_{i}^{w_{0}} \sim \iota_{s_{1}}^{w_{0}} \circ \iota_{w_{0}}^{s_{1}} \circ \iota_{i}^{w_{0}} \sim p^{2} \iota_{i}^{w_{0}}$ by Proposition 2.2.4. and so $\iota_{j}^{i} \circ \iota_{i}^{j}=p^{2}$.

For (3), by relabelling $a, b$, and $c$, we can assume without loss of generality that $s=e$. From Propositions 2.2.2 and 2.2.6 we see that $\iota_{e}^{i} \sim \iota_{s_{1}}^{i} \circ \iota_{e}^{s_{1}}$ and $\iota_{i}^{e} \sim \iota_{s_{1}}^{e} \circ \iota_{i}^{s_{1}}$. Hence $\iota_{i}^{e} \circ \iota_{e}^{i} \sim \iota_{s_{1}}^{e} \circ \iota_{i}^{s_{1}} \circ \iota_{s_{1}}^{i} \circ \iota_{e}^{s_{1}}=p^{2}$ by Proposition 2.2.4 and (2).

2.3. Lattices via Morita theory. In this subsection, we use Morita theory to explicitly describe the moduli of lattices in a given residually multiplicity free $K$ representation. The idea to use Morita theory to describe the moduli of lattices was suggested by David Helm (see [EGS15, §1.6]).

2.3.1. The abelian category generated by lattices. Let $\tau$ be a continuous irreducible (finite dimensional) $K$-representation over $\mathbf{Q}_{p}$, which is residually multiplicity free. Let $\mathcal{L}$ be the category of all finitely generated $\mathbf{Z}_{p}$-modules with a $K$-action which are 
isomorphic to subquotients of $\mathbf{Z}_{p}$-lattices in $\tau^{\oplus n}$ for some $n \in \mathbf{N}$. The irreducible objects of $\mathcal{L}$ are $\sigma$ where $\sigma$ is a Jordan-Hölder factor of $\bar{\tau}$. Let $\tau^{\sigma} \subset \tau$ be a lattice (unique up to homothety by [EGS15, Lemma 4.1.1]) with cosocle $\sigma$.

Let $\Gamma$ be the group $\mathrm{GL}_{3}\left(\mathbf{F}_{p}\right)$. Let $P^{\sigma} \rightarrow \sigma$ be a projective envelope in the category of $\mathbf{Z}_{p}[\Gamma]$-modules. Then there exists a surjection $\varphi: P^{\sigma} \rightarrow \tau^{\sigma}$, which we fix. The following lemma appears in the proof of [EGS15, Proposition 4.2.1].

Lemma 2.3.1. The space $\operatorname{Hom}_{\mathbf{Z}_{p}[\Gamma]}\left(P^{\sigma}, \tau^{\sigma}\right)$ is a free $\mathbf{Z}_{p}$-module generated by $\varphi$.

Proof. The space is clearly free over $\mathbf{Z}_{p}$, being finitely generated and torsion-free. By projectivity of $P^{\sigma}$, we have

$$
\operatorname{Hom}_{\mathbf{Z}_{p}[\Gamma]}\left(P^{\sigma}, \tau^{\sigma}\right) / p \operatorname{Hom}_{\mathbf{Z}_{p}[\Gamma]}\left(P^{\sigma}, \tau^{\sigma}\right) \cong \operatorname{Hom}_{\mathbf{F}_{p}[\Gamma]}\left(\bar{P}^{\sigma}, \bar{\tau}^{\sigma}\right) \cong \operatorname{Hom}_{\mathbf{F}_{p}[\Gamma]}\left(\bar{P}^{\sigma}, \bar{\tau}^{\mathrm{ss}}\right),
$$

where the last isomorphism follows from projectivity of $\bar{P}^{\sigma}$ as an $\mathbf{F}_{p}[\Gamma]$-module. Since $\operatorname{Hom}_{\mathbf{F}_{p}[\Gamma]}\left(\bar{P}^{\sigma}, \bar{\tau}^{\mathrm{ss}}\right)$ is one-dimensional and generated by the image of $\varphi$, the result now follows from Nakayama's lemma.

Lemma 2.3.2. For any $M \in \mathcal{L}$, any $\mathbf{Z}_{p}[\Gamma]$-homomorphism $P^{\sigma} \rightarrow M$ factors through $\varphi$.

Proof. Suppose that $M$ is a quotient of a lattice $N \subset \tau^{\oplus n}$. Then the map $P^{\sigma} \rightarrow M$ lifts to a map $P^{\sigma} \rightarrow N$. For some embedding $\tau^{\sigma} \subset \tau$, we have $N \subset\left(\tau^{\sigma}\right)^{\oplus n}$. So we can assume without loss of generality that $M=\left(\tau^{\sigma}\right)^{\oplus n}$. Since by Lemma 2.3.1 $\operatorname{Hom}_{\mathbf{Z}_{p}[\Gamma]}\left(P,\left(\tau^{\sigma}\right)^{\oplus n}\right)$ is generated by maps which factor through $\varphi$, any such map factors through $\varphi$.

Proposition 2.3.3. The lattices $\tau^{\sigma}$ are projective objects in $\mathcal{L}$.

Proof. We show that $\tau^{\sigma}$ satisfies the lifting property. Let $M \rightarrow N$ be a surjection in $\mathcal{L}$. We wish to lift a map $\tau^{\sigma} \rightarrow N$ to a map $\tau^{\sigma} \rightarrow M$. The composition $P^{\sigma} \rightarrow \tau^{\sigma} \rightarrow N$ with $\varphi$ lifts to $P^{\sigma} \rightarrow M$ by projectivity of $P^{\sigma}$, which factors through $\varphi$ by Lemma 2.3.2. Since $\varphi$ is surjective, this gives the required lifting.

Let $\mathcal{P}=\oplus_{\sigma} \tau^{\sigma}$ where $\sigma$ runs over the distinct Jordan-Hölder factors of $\bar{\tau}$. Let $\mathcal{E}=\operatorname{End}_{K}(\mathcal{P})$.

Proposition 2.3.4. The functor $M \mapsto \operatorname{Hom}_{K}(\mathcal{P}, M)$ gives an equivalence of categories $\mathcal{L} \rightarrow$ f.g. $\mathcal{E}^{o p}$-modules.

Proof. By Proposition 2.3.3 $\mathcal{P}$ is projective generator of the category $\mathcal{L}$. The equivalence follows from Morita theory with quasi-inverse given by $(\cdot) \otimes_{\mathcal{E}^{\circ \mathrm{p}}} \mathcal{P}$.

2.3.2. Moduli of lattices in algebraic vectors. We use the notation $V, W$, and $W^{0}$ from 2.1. Let $\mathcal{E}^{\text {alg }}:=\operatorname{End}_{K}\left(W^{0} \oplus W\right)$. Using Proposition 2.1.2 it is easy to calculate that there is an isomorphism

$$
\mathcal{E}^{\text {alg }} \stackrel{\sim}{\rightarrow}\left(\begin{array}{cc}
\mathbf{Z}_{p} & p \mathbf{Z}_{p} \\
\mathbf{Z}_{p} & \mathbf{Z}_{p}
\end{array}\right)
$$

where $\left(\begin{array}{ll}1 & 0 \\ 0 & 0\end{array}\right)$ and $\left(\begin{array}{ll}0 & 0 \\ 0 & 1\end{array}\right)$ restrict to the identity on $W^{0}$ and $W$, respectively, and $\left(\begin{array}{ll}0 & p \\ 0 & 0\end{array}\right)$ and $\left(\begin{array}{ll}0 & 0 \\ 1 & 0\end{array}\right)$ correspond to $i^{\vee}$ and $i$, respectively. 
We now use Proposition 2.3.4 to describe the moduli space of lattices in $V$ (with rigidifications). Let $\mathcal{M}$ be the functor which takes a $\mathbf{Z}_{p}$-algebra $R$ to the set

$$
\begin{array}{r}
\left\{\left(\mathcal{P}_{R}, \psi_{1}, \psi_{2}\right) \mid \mathcal{P}_{R} \text { is a free } R \text {-module with an } \mathcal{E}^{\text {alg }}\right. \text {-action, } \\
\left.\psi_{1}: R \stackrel{\sim}{\rightarrow}\left(\begin{array}{ll}
1 & 0 \\
0 & 0
\end{array}\right) \mathcal{P}_{R}, \psi_{2}: R \stackrel{\sim}{\rightarrow}\left(\begin{array}{ll}
0 & 0 \\
0 & 1
\end{array}\right) \mathcal{P}_{R}\right\} / \cong
\end{array}
$$

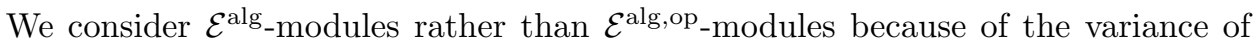
the patching construction in 4 .

Proposition 2.3.5. The functor $\mathcal{M}$ is represented by the ring $\mathbf{Z}_{p}[x, y] /(x y-p)$ where the map $\mathbf{Z}_{p}[x, y] /(x y-p) \rightarrow R$ classifying $\left(\mathcal{P}_{R}, \psi_{1}, \psi_{2}\right)$ is given by $x \mapsto$ $\psi_{1}^{-1} \circ\left(\begin{array}{cc}0 & p \\ 0 & 0\end{array}\right) \circ \psi_{2}$ and $y \mapsto \psi_{2}^{-1} \circ\left(\begin{array}{ll}0 & 0 \\ 1 & 0\end{array}\right) \circ \psi_{1}$.

Proof. Let $A=\mathbf{Z}_{p}[x, y] /(x y-p)$. Then we define $\mathcal{P}_{A}$ to be $A^{2}$ where $\mathcal{E}^{\text {alg }} \rightarrow M_{2}(A)$ is given by

$$
\begin{aligned}
& \left(\begin{array}{ll}
1 & 0 \\
0 & 0
\end{array}\right) \mapsto\left(\begin{array}{ll}
1 & 0 \\
0 & 0
\end{array}\right) \\
& \left(\begin{array}{ll}
0 & 0 \\
0 & 1
\end{array}\right) \mapsto\left(\begin{array}{ll}
0 & 0 \\
0 & 1
\end{array}\right) \\
& \left(\begin{array}{ll}
0 & p \\
0 & 0
\end{array}\right) \mapsto\left(\begin{array}{ll}
0 & x \\
0 & 0
\end{array}\right) \\
& \left(\begin{array}{ll}
0 & 0 \\
1 & 0
\end{array}\right) \mapsto\left(\begin{array}{ll}
0 & 0 \\
y & 0
\end{array}\right)
\end{aligned}
$$

The triple $\left(P_{A}, \mathrm{id}_{A}, \mathrm{id}_{A}\right)$ is universal since $\psi_{1} \oplus \psi_{2}:\left(R^{2}, \operatorname{id}_{R}, \operatorname{id}_{R}\right) \rightarrow\left(\mathcal{P}_{R}, \psi_{1}, \psi_{2}\right)$ is an isomorphism. Here the $\mathcal{E}^{\text {alg }}$-action of $R^{2}$ is given by the map $A \rightarrow R$ described in the statement of the proposition.

\section{HeCke ALGEBRas}

In this section, we describe integral Hecke operators for some locally algebraic types, and relate them to maps between lattices. We give some general facts about Hecke algebras in 3.1 that we specialize to extensions of weights in $\$ 3.2$ and to principal series types in 3.3 .

3.1. Hecke algebras for split reductive groups. We first describe integral Hecke algebras for general representations of the $\mathbf{Z}_{p}$-points of split reductive groups. Let $\mathbf{G}$ be a split reductive group over $\mathbf{Z}_{p}$, and $\mathbf{T} \subset \mathbf{B}$ a maximal torus and a Borel subgroup. Let $X_{*}(\mathbf{T})$ (resp. $X_{*}(\mathbf{T})_{-}$) denote the group of cocharacters (resp. antidominant cocharacters) of $\mathbf{T}$. Let $K=\mathbf{G}\left(\mathbf{Z}_{p}\right)$ be a maximal compact open hyperspecial subgroup of $G=\mathbf{G}\left(\mathbf{Q}_{p}\right)$ and

$$
K_{1}=\operatorname{ker}\left(\mathbf{G}\left(\mathbf{Z}_{p}\right) \rightarrow \mathbf{G}\left(\mathbf{F}_{p}\right)\right) .
$$

For $K$-representations $W_{1}$, and $W_{2}$ over $\mathbf{Z}_{p}$, define the Hecke bimodule

$$
\mathcal{H}\left(W_{1}, W_{2}\right):=\operatorname{Hom}_{G}\left(\operatorname{ind}_{K}^{G} W_{1}, \operatorname{ind}_{K}^{G} W_{2}\right),
$$

which by Frobenius reciprocity is isomorphic to the space of compactly supported functions $f: G \rightarrow \operatorname{Hom}_{\mathbf{Z}_{p}}\left(W_{1}, W_{2}\right)$ such that $f\left(k_{2} g k_{1}\right)=k_{2} \circ f(g) \circ k_{1}$ for all $k_{1}, k_{2} \in K$ and $g \in G$. If $W$ is a $K$-representation, let $\mathcal{H}(W)=\mathcal{H}(W, W)$ be the Hecke algebra of $W$. If $\pi$ is a $G$-representation over $\mathbf{Z}_{p}$, then $\operatorname{Hom}_{K}(W, \pi)$ is a right $\mathcal{H}_{G}(W)$-module.

Recall the Cartan decomposition

$$
G=\sqcup_{\mu \in X_{*}(\mathbf{T})_{-}} K t_{\mu} K
$$


where $t_{\mu}=\mu(p)$. This gives the decomposition

$$
\operatorname{ind}_{K}^{G} W=\bigoplus_{\mu \in X_{*}(\mathbf{T})_{-}} \operatorname{Ind}_{K}^{K t_{\mu} K} W
$$

as $K$-representations where

$$
\operatorname{Ind}_{K}^{K t K} W=\{f: K t K \rightarrow W \mid f(k x)=k f(x) \forall k \in K, x \in K t K\}
$$

and the $K$-action is the right regular action. We can simplify this as follows:

$$
\operatorname{Ind}_{K}^{K t K} W \cong \operatorname{Ind}_{K \cap t K t^{-1}}^{t K} W \cong \operatorname{Ind}_{t^{-1} K t \cap K}^{K} W^{(t)}
$$

where $W^{(t)}$ is identified with $W$ as vector spaces, but the superscript denotes the twisted action on $W^{(t)}$ defined by $k w^{(t)}=\left(t k t^{-1} w\right)^{(t)}$ for $w^{(t)} \in W^{(t)}$ and $k \in$ $t^{-1} K t$.

Given a coweight $\mu \in X_{*}(\mathbf{T})_{-}$, let $\mathcal{H}\left(W_{1}, W_{2}\right)_{\mu}=\operatorname{Hom}_{K}\left(W_{1}, \operatorname{Ind}_{K}^{K t_{\mu} K} W_{2}\right) \subset$ $\mathcal{H}\left(W_{1}, W_{2}\right)$ denote the subspace of elements supported on the double coset $K t_{\mu} K$. Let $\widetilde{N}_{\mu}$ and $\widetilde{P}_{\mu}$ be $t_{\mu}^{-1} K_{1} t_{\mu} \cap K$ and $t_{\mu}^{-1} K t_{\mu} \cap K$, respectively. The following proposition is contained in the proof of [Her11b, Theorem 1.2].

Proposition 3.1.1. Suppose that $K_{1}$ acts trivially on $W_{1}$ and $W_{2}$. We have a natural isomorphism $\mathcal{H}\left(W_{1}, W_{2}\right)_{\mu} \cong \operatorname{Hom}_{\widetilde{P}_{\mu}}\left(\left(W_{1}\right)_{\widetilde{N}_{\mu}},\left(W_{2}^{\widetilde{N}_{-\mu}}\right)^{\left(t_{\mu}\right)}\right)$.

Proof. We have a natural injection

$$
\begin{aligned}
\operatorname{Hom}_{\widetilde{P}_{\mu}}\left(\left(W_{1}\right)_{\widetilde{N}_{\mu}},\left(W_{2}^{\widetilde{N}_{-\mu}}\right)^{\left(t_{\mu}\right)}\right) \hookrightarrow \operatorname{Hom}_{\widetilde{P}_{\mu}}\left(W_{1}, W_{2}^{\left(t_{\mu}\right)}\right) & \cong \operatorname{Hom}_{K}\left(W_{1}, \operatorname{Ind}_{\widetilde{P}_{\mu}}^{K} W_{2}^{\left(t_{\mu}\right)}\right) \\
& \cong \mathcal{H}\left(W_{1}, W_{2}\right)_{\mu}
\end{aligned}
$$

where the first isomorphism follows from Frobenius reciprocity and the second isomorphism follows from (3.1.2). It suffices to show surjectivity of the first map. Let $f \in \operatorname{Hom}_{\widetilde{P}_{\mu}}\left(W_{1}, W_{2}^{\left(t_{\mu}\right)}\right)$. Since $W_{1}$ is $K_{1}$-invariant, the image of $f$ is contained in

$$
\left(W_{2}^{\left(t_{\mu}\right)}\right)^{K_{1} \cap t_{\mu}^{-1} K t_{\mu}}=\left(W_{2}^{t_{\mu} K_{1} t_{\mu}^{-1} \cap K}\right)^{\left(t_{\mu}\right)}=\left(W_{2}^{\widetilde{N}_{-\mu}}\right)^{\left(t_{\mu}\right)} .
$$

Similarly, since $W_{2}$ is $K_{1}$-invariant, $W_{2}^{\left(t_{\mu}\right)}$ is $\widetilde{N}_{\mu}$-invariant. Therefore the map $f$ factors through $\left(W_{1}\right)_{\widetilde{N}_{\mu}}$.

We can rephrase Proposition 3.1.1 as follows. Given a $\widetilde{P}_{\mu}$-morphism $\left(W_{1}\right)_{\widetilde{N}_{\mu}} \rightarrow$ $\left(W_{2}^{\widetilde{N}_{-\mu}}\right)^{\left(t_{\mu}\right)}$, we obtain a $K$-morphism $\operatorname{Ind}_{\widetilde{P}_{\mu}}^{K}\left(W_{1}\right)_{\widetilde{N}_{\mu}} \rightarrow \operatorname{Ind}_{\widetilde{P}_{\mu}}^{K}\left(W_{2}^{\widetilde{N}_{-\mu}}\right)^{\left(t_{\mu}\right)}$. By Frobenius reciprocity, we have a natural map $W_{1} \rightarrow \operatorname{Ind}_{\widetilde{P}_{\mu}}^{K}\left(W_{1}\right)_{\widetilde{N}_{\mu}}$. The composition

$$
W_{1} \rightarrow \operatorname{Ind}_{\widetilde{P}_{\mu}}^{K}\left(W_{1}\right)_{\widetilde{N}_{\mu}} \rightarrow \operatorname{Ind}_{\widetilde{P}_{\mu}}^{K}\left(W_{2}^{\widetilde{N}_{-\mu}}\right)^{\left(t_{\mu}\right)} \subset \operatorname{Ind}_{\widetilde{P}_{\mu}}^{K} W_{2}^{\left(t_{\mu}\right)} \cong \operatorname{Ind}_{K}^{K t_{\mu} K} W_{2}
$$

is the corresponding element of $\mathcal{H}\left(W_{1}, W_{2}\right)_{\mu}$.

We can further simplify this when $\mu$ is minuscule, which we now suppose. See $\S 3$ and particularly [Her11b, Proposition 3.8] for a more general context. Let $N_{\mu}$ and $P_{\mu}$ be the images of $\widetilde{N}_{\mu}$ and $\widetilde{P}_{\mu}$ in $\mathbf{G}\left(\mathbf{F}_{p}\right)$, respectively. Then $N_{\mu}$ and $P_{\mu}$ are the usual unipotent and parabolic subgroups in $\mathbf{G}\left(\mathbf{F}_{p}\right)$, respectively, corresponding to $\mu$. Let $M_{\mu}=P_{\mu} / N_{\mu}$. Note that $t_{\mu} \widetilde{P}_{\mu} t_{\mu}^{-1}=\widetilde{P}_{-\mu}$ and $t_{\mu} \widetilde{N}_{\mu} K_{1} t_{\mu}^{-1}=\widetilde{N}_{-\mu} K_{1}$, and so conjugation by $t_{\mu}$ gives an isomorphism $M_{\mu} \cong M_{-\mu}$. 
Proposition 3.1.2. Suppose that $\mu$ is minuscule and that $K_{1}$ acts trivially on $W_{1}$ and $W_{2}$. We have a natural isomorphism $\mathcal{H}\left(W_{1}, W_{2}\right)_{\mu} \cong \operatorname{Hom}_{M_{\mu}}\left(\left(W_{1}\right)_{N_{\mu}}, W_{2}^{N_{-\mu}}\right)$ where $M_{\mu}$ acts on $W_{2}^{N_{-\mu}}$ through the isomorphism $M_{\mu} \stackrel{\sim}{\rightarrow} M_{-\mu}$ given by conjugation by $t_{\mu}$.

Proof. Since $\mu$ is minuscule, $K_{1} \subset \widetilde{P}_{\mu}$. Using Proposition 3.1.1 we have

$$
\begin{aligned}
\mathcal{H}\left(W_{1}, W_{2}\right)_{\mu} & \cong \operatorname{Hom}_{\widetilde{P}_{\mu}}\left(\left(W_{1}\right)_{\widetilde{N}_{\mu}},\left(W_{2}^{\widetilde{N}_{-\mu}}\right)^{\left(t_{\mu}\right)}\right) \\
& \cong \operatorname{Hom}_{\widetilde{P}_{\mu}}\left(\left(W_{1}\right)_{\widetilde{N}_{\mu} K_{1}},\left(W_{2}^{\widetilde{N}_{-\mu} K_{1}}\right)^{\left(t_{\mu}\right)}\right) \\
& \cong \operatorname{Hom}_{M_{\mu}}\left(\left(W_{1}\right)_{N_{\mu}}, W_{2}^{N_{-\mu}}\right) .
\end{aligned}
$$

We specialize the above discussion to the case when $W_{1}=W_{2}=\sigma$ is an irreducible $\mathbf{G}\left(\mathbf{F}_{p}\right)$-representation over $\mathbf{F}_{p}$ (and hence a $K$-representation by inflation) and $\mu$ is a minuscule coweight. By [Her11a, Lemma 2.5], $\sigma_{N_{\mu}}$ is irreducible, and the composition of canonical maps $\sigma^{N_{-\mu}} \subset \sigma \rightarrow \sigma_{N_{\mu}}$ is an isomorphism of $M_{\mu} \cong M_{-\mu}$-representations. Recalling that the isomorphism $M_{\mu} \cong M_{-\mu}$ is given by $t_{\mu}$-conjugation, we get a $\widetilde{P}_{\mu}$-isomorphism $\sigma_{N_{\mu}} \stackrel{\sim}{\rightarrow} t_{-\mu} \cdot \sigma^{N_{-\mu}} \subset \operatorname{ind}_{\widetilde{P}_{-\mu}^{G}}^{G} \sigma^{N_{-\mu}}$. This composite induces a nonzero map

$$
T_{\sigma, \mu}^{\prime}: \operatorname{ind}_{\widetilde{P}_{\mu}}^{G} \sigma_{N_{\mu}} \rightarrow \operatorname{ind}_{\widetilde{P}_{-\mu}}^{G} \sigma^{N_{-\mu}} .
$$

As $\mathcal{H}(\sigma)_{\mu}$ is one-dimensional by loc. cit. and Proposition 3.1.2, the composition of $T_{\sigma, \mu}^{\prime}$ with the natural inclusion $\operatorname{ind}_{\widetilde{P}_{-\mu}}^{G} \sigma^{N_{-\mu}} \cong \operatorname{ind}_{\widetilde{P}_{\mu}}^{G}\left(\sigma^{\left.N_{-\mu}\right)^{\left(t_{\mu}\right)}} \subset \operatorname{ind}_{K}^{K t_{\mu} K} \sigma\right.$ is a generator of $\mathcal{H}(\sigma)_{\mu}$. The following is a rephrasing of weight cycling as in [EGH13, Proposition 2.3.1].

Proposition 3.1.3. The map (3.1.3) is an isomorphism.

Proof. Symmetrically to the definition of $T_{\sigma, \mu}^{\prime}$, one can define $\widetilde{P}_{-\mu}$-isomorphism $\sigma^{N_{-\mu}} \stackrel{\sim}{\rightarrow} t_{\mu} \cdot \sigma_{N_{\mu}} \subset \operatorname{ind}_{\widetilde{P}_{\mu}}^{G} \sigma_{N_{\mu}}$. The composite induces a map $\operatorname{ind}_{\widetilde{P}_{-\mu}}^{G} \sigma^{N_{-\mu}} \rightarrow$ $\operatorname{ind} \underset{\widetilde{P}_{\mu}}{G} \sigma_{N_{\mu}}$ which is the inverse of $T_{\sigma, \mu}^{\prime}$.

We omit $\mu$ from the notation if it is clear from context. The following proposition follows from the discussion after Proposition 3.1.1.

Proposition 3.1.4. If $T^{\prime} \in \mathcal{H}\left(W_{1}, W_{2}\right)_{\mu}$ corresponds via Proposition 3.1.2 to a map that factors through $\sigma_{N_{\mu}} \cong \sigma^{N_{-\mu}}$, then $T^{\prime}$ factors through $T_{\sigma, \mu}^{\prime}$ as $\operatorname{ind}_{K}^{G} W_{1} \rightarrow$ $\operatorname{ind}_{K}^{G} \operatorname{Ind}_{\widetilde{P}_{\mu}}^{K} \sigma_{N_{\mu}} \rightarrow \operatorname{ind}_{K}^{G} \operatorname{Ind}_{\widetilde{P}_{-\mu}}^{K} \sigma^{N_{-\mu}} \rightarrow \operatorname{ind}_{K}^{G} W_{2}$.

3.2. Hecke algebras of extensions for $\mathrm{GL}_{3}$. We specialize 3.1 to the case of $\mathbf{G}=\mathrm{GL}_{3}$ and certain extensions of weights, extending some of the results in Her11a. Let $\mu$ be an antidominant minuscule coweight. If $\mu$ is $(0,0,1)$ (resp. $(0,1,1)$ ), then $N_{\mu}=\bar{N}_{1}$ (resp. $\bar{N}_{2}$ ) in the notation of $\$ 2.2 .1$ Let $a, b$, and $c$ be integers such that $a>b>c$ and $a-c<p-1$. Let $\sigma^{\prime}$ be the upper alcove Serre weight $F(c+p-1, b, a-p+1)$ so that $\sigma_{N_{\mu}}^{\prime} \cong F(b+p-1, a) \otimes F(c)$ or $F(a) \otimes F(c, b-p+1)$. Let $\sigma$ be $F(a-1, b, c+1)$. Recall that we defined $\bar{W}$ and $\bar{W}^{0}$ in $\$ 2.1$ Then $\bar{W}$ is a nonsplit extension of $\sigma$ by $\sigma^{\prime}$ and $\bar{W}^{0}$ is a nonsplit extension of $\sigma^{\prime}$ by $\sigma$. Note that there is an injection $\bar{W} \hookrightarrow \operatorname{Ind}_{\widetilde{P}_{\mu}}^{K} \sigma_{N_{\mu}}^{\prime}=\operatorname{Ind}_{P_{\mu}}^{\mathrm{GL}_{3}\left(\mathbf{F}_{p}\right)} \sigma_{N_{\mu}}^{\prime}$. 
Proposition 3.2.1. We have $\bar{W}_{N_{\mu}} \cong \sigma_{N_{\mu}} \oplus \sigma_{N_{\mu}}^{\prime}, \bar{W}^{N_{-\mu}} \cong\left(\sigma^{\prime}\right)^{N_{-\mu}}, \bar{W}_{N_{\mu}}^{0} \cong \sigma_{N_{\mu}}^{\prime}$, and $\left(\bar{W}^{0}\right)^{N_{-\mu}} \cong \sigma^{N_{-\mu}} \oplus\left(\sigma^{\prime}\right)^{N_{-\mu}}$. The spaces $\mathcal{H}(\bar{W})_{\mu}$ and $\mathcal{H}\left(\bar{W}^{0}\right)_{\mu}$ are both onedimensional over $\mathbf{F}_{p}$.

Proof. We have exact sequences

$$
\begin{aligned}
0 \rightarrow\left(\sigma^{\prime}\right)^{N_{-\mu}} & \rightarrow \bar{W}^{N_{-\mu}} \rightarrow \sigma^{N_{-\mu}} \\
0 \rightarrow \sigma^{N_{-\mu}} & \rightarrow\left(\bar{W}^{0}\right)^{N_{-\mu}} \rightarrow\left(\sigma^{\prime}\right)^{N_{-\mu}} \\
\sigma_{N_{\mu}}^{\prime} & \rightarrow \bar{W}_{N_{\mu}} \rightarrow \sigma_{N_{\mu}} \rightarrow 0 \\
\sigma_{N_{\mu}} & \rightarrow \bar{W}_{N_{\mu}}^{0} \rightarrow \sigma_{N_{\mu}}^{\prime} \rightarrow 0 .
\end{aligned}
$$

By Her11a, Lemma 2.5] and the fact that $c \not \equiv a-1(\bmod p-1)$, the irreducible $M_{\mu^{-}}$ representations $\left(\sigma^{\prime}\right)^{N_{-\mu}} \cong \sigma_{N_{\mu}}^{\prime}$ and $\sigma^{N_{-\mu}} \cong \sigma_{N_{\mu}}$ have different actions of the $\mathrm{GL}_{1}$ factor of $M_{\mu}$. Thus, if the above exact sequences extend to short exact sequences, they must split. It suffices then to determine whether these exact sequences extend to short exact sequences.

Since $\sigma^{\prime}$ is not a Jordan-Hölder factor of $\operatorname{Ind}_{P_{-\mu}}^{\mathrm{GL}_{3}\left(\mathbf{F}_{p}\right)} \sigma^{N_{-\mu}}\left(\operatorname{resp} . \operatorname{Ind}_{P_{\mu}}^{\mathrm{GL}_{3}\left(\mathbf{F}_{p}\right)} \sigma_{N_{\mu}}\right)$ as can be checked casewise using [EGH13, Lemma 6.1.1], any map $\operatorname{Ind}_{P_{-\mu}}^{\mathrm{GL}_{3}\left(\mathbf{F}_{p}\right)} \sigma^{N_{-\mu}} \rightarrow$ $\bar{W}$ (resp. $\bar{W}^{0} \rightarrow \operatorname{Ind}_{P_{\mu}}^{\mathrm{GL}_{3}\left(\mathbf{F}_{p}\right)} \sigma_{N_{\mu}}$ ) must be zero. By Frobenius reciprocity, we conclude that $\bar{W}^{N_{-\mu}} \cong\left(\sigma^{\prime}\right)^{N_{-\mu}}$ and $\bar{W}_{N_{\mu}}^{0} \cong \sigma_{N_{\mu}}^{\prime}$.

On the other hand, we have an inclusion $\bar{W} \subset \operatorname{Ind}_{P_{\mu}}^{\mathrm{GL}_{3}\left(\mathbf{F}_{p}\right)} \sigma_{N_{\mu}}^{\prime}$. By Frobenius reciprocity, we conclude that $\bar{W}_{N_{\mu}} \cong \sigma_{N_{\mu}} \oplus \sigma_{N_{\mu}}^{\prime}$. We claim that there exists a surjection $\operatorname{Ind}_{P_{-\mu}}^{\mathrm{GL}_{3}\left(\mathbf{F}_{p}\right)}\left(\sigma^{\prime}\right)^{N_{-\mu}} \rightarrow \bar{W}^{0}$, as can be checked from Proposition 2.2.1. By Frobenius reciprocity, we conclude that $\left(\bar{W}^{0}\right)^{N_{-\mu}} \cong \sigma^{N_{-\mu}} \oplus\left(\sigma^{\prime}\right)^{N_{-\mu}}$. The second part now follows from Proposition 3.1 .2

Remark 3.2.2. An analogous result (with some hypotheses on $a, b$, and $c$ ) could be proven for $\sigma$ one of the weights $F(c+p-2, a, b+1)$ and $F(b-1, c, a-p+2)$ and $\bar{W}$ replaced by the extension of $\sigma$ by $\sigma^{\prime}$ and $\bar{W}^{0}$ similarly replaced, but we will not use this in what follows.

Proposition 3.2.3. The standard $G$-action on $V$ gives an isomorphism $\operatorname{ind}_{K}^{G} V \cong$ $V \otimes \operatorname{ind}_{K}^{G} \mathbf{1}$ which induces a canonical isomorphism $\mathcal{H}(V)_{\mu} \cong \mathcal{H}(\mathbf{1})_{\mu}$. For an arbitrary $\mu \in X_{*}(\mathbf{T})_{-}, \mathcal{H}\left(W, W_{0}\right)_{\mu}, \mathcal{H}\left(W^{0}\right)_{\mu}$, and $\mathcal{H}(W)_{\mu}$ are canonically lattices in the one dimensional space $\mathcal{H}(V)_{\mu} \cong \mathcal{H}(\mathbf{1})_{\mu}$ via $i: W_{0} \hookrightarrow W \subset V$. Moreover, the double coset operator $p^{\left\langle\mu, w_{0} \nu\right\rangle} K t_{\mu} K$ lies in all these lattices.

Proof. The isomorphism $\mathcal{H}(V)_{\mu} \cong \mathcal{H}(\mathbf{1})_{\mu}$ comes from the fact that $V$ is irreducible. The space $\mathcal{H}(\mathbf{1})_{\mu}$ is one-dimensional over $\mathbf{Q}_{p}$ by the Satake isomorphism. Since $t_{\mu}$ acts on the $\lambda$-weight space of $V^{*}$ by $p^{\langle\mu, \lambda\rangle}$ and $-w_{0} \nu$ is the highest weight of $V^{*}$, $p^{\left\langle\mu, w_{0} \nu\right\rangle} K t_{\mu} K$ stabilizes

$$
\mathcal{H}(W) \cong \operatorname{Hom}_{K}\left(W, \operatorname{ind}_{K}^{G} W\right) \cong\left(W^{d} \otimes \operatorname{ind}_{K}^{G} W\right)^{K} \subset V^{*} \otimes \operatorname{ind}_{K}^{G} V
$$

and thus is the image of an element in $\mathcal{H}(W)_{\mu}$. (Recall that $W^{d}$ is the Schikhof or $\mathbf{Z}_{p^{-}}$-dual of $W$.) The proof for the other lattices are the same. 
The reduction $i: \bar{W}^{0} \rightarrow \bar{W}$ factors through a map $\sigma^{\prime} \hookrightarrow \bar{W}$ which we fix. Let $T^{\prime} \in \mathcal{H}\left(W, W^{0}\right)_{\mu}$ be the element which maps to the double coset operator $p^{\left\langle\mu, w_{0} \nu\right\rangle} K t_{\mu} K$ in Proposition 3.2 .3

Proposition 3.2.4. The inclusion $i$ induces natural maps $\mathcal{H}\left(W, W^{0}\right)_{\mu} \rightarrow \mathcal{H}\left(W^{0}\right)_{\mu}$ and $\mathcal{H}\left(W, W^{0}\right)_{\mu} \rightarrow \mathcal{H}(W)_{\mu}$, which are isomorphisms between rank one $\mathbf{Z}_{p}$-modules. Furthermore, the map $\mathcal{H}\left(\bar{W}, \bar{W}^{0}\right)_{\mu} \rightarrow \mathcal{H}\left(\sigma^{\prime}\right)_{\mu}$, induced by the fixed maps $\sigma^{\prime} \hookrightarrow \bar{W}$ and $i$, is an isomorphism. Finally, $T^{\prime}$ generates $\mathcal{H}\left(W, W^{0}\right)_{\mu}$ over $\mathbf{Z}_{p}$.

Proof. As in the proof of Proposition 3.2.3. $p^{\left\langle\mu, w_{0} \nu\right\rangle} t_{\mu}$ stabilizes on $W^{d}$. Mod $p$, it acts by zero on all weight spaces except for the $-w_{0} \nu$-weight space. Let $h$ be the natural isomorphism from $\mathcal{H}\left(\sigma^{\prime}\right)$ to the space of compactly supported functions $f: G \rightarrow \operatorname{End}_{K}\left(\sigma^{\prime}\right)$ satisfying $f\left(k_{1} g k_{2}\right)=k_{1} \circ f(g) \circ k_{2}$. If $T$ is the image of $T^{\prime}$ in $\mathcal{H}(W)$, then the reduction of $T \bmod p$ stabilizes $\operatorname{ind}_{K}^{G} \sigma^{\prime}$ and $h(T)(p)$ is the projection to the $w_{0} \nu$-weight space. In particular, the map $\mathcal{H}\left(\bar{W}, \bar{W}^{0}\right)_{\mu} \rightarrow \mathcal{H}\left(\sigma^{\prime}\right)_{\mu}$ is nonzero and thus an isomorphism since the domain and codomain are both onedimensional by Proposition 3.2.1. Furthermore, the maps $\mathcal{H}\left(\bar{W}, \bar{W}^{0}\right)_{\mu} \rightarrow \mathcal{H}(\bar{W})_{\mu}$ and $\mathcal{H}\left(\bar{W}, \bar{W}^{0}\right)_{\mu} \rightarrow \mathcal{H}\left(\bar{W}^{0}\right)_{\mu}$ induced by $i$ are isomorphisms. By Nakayama's lemma, the maps $\mathcal{H}\left(W, W^{0}\right)_{\mu} \rightarrow \mathcal{H}(W)_{\mu}$ and $\mathcal{H}\left(W, W^{0}\right)_{\mu} \rightarrow \mathcal{H}\left(W^{0}\right)_{\mu}$ induced by $i$ are isomorphisms and $T^{\prime}$ generates $\mathcal{H}\left(W, W^{0}\right)_{\mu}$ over $\mathbf{Z}_{p}$.

By abuse of notation, let $T$ denote the generator which is the image of $T^{\prime}$ in $\mathcal{H}(W)_{\mu}, \mathcal{H}(\bar{W})_{\mu}, \mathcal{H}\left(W^{0}\right)_{\mu}, \mathcal{H}\left(\bar{W}^{0}\right)_{\mu}$, and $\mathcal{H}\left(\sigma^{\prime}\right)_{\mu}$ via the maps in Proposition 3.2.4 We record this in the following proposition.

Proposition 3.2.5. The morphism $T \in \mathcal{H}\left(W^{0}\right)_{\mu}$ factors as $\operatorname{ind}_{K}^{G} W^{0} \stackrel{i}{\rightarrow} \operatorname{ind}_{K}^{G} W \stackrel{T^{\prime}}{\rightarrow}$ $\operatorname{ind}_{K}^{G} W^{0}$.

3.3. Hecke algebras for principal series types. In this section, we describe Hecke operators for principal series types. We use the notation of $\$ 2.2 .2$, with the following abuses of notation: $v^{s}$ also denotes the image of $v^{s} \in \operatorname{ind}_{I}^{K} \chi^{s}$ under the natural map $\operatorname{ind}_{I}^{K} \chi^{s} \rightarrow \operatorname{ind}_{I}^{G} \chi^{s}$, and $\iota_{s^{\prime}}^{s}$ also denotes $\operatorname{ind}_{K}^{G}\left(\iota_{s^{\prime}}^{s}\right)$. Let $t_{1}=\left(\begin{array}{ccc}p^{-1} & 0 & 0 \\ 0 & 1 & 0 \\ 0 & 0 & 1\end{array}\right)$ and $t_{2}=\left(\begin{array}{ccc}p^{-1} & 0 & 0 \\ 0 & p^{-1} & 0 \\ 0 & 0 & 1\end{array}\right)$. Let $(i, j)$ be a permutation of $(1,2)$.

Proposition 3.3.1. The element $r_{i} t_{i} v^{s}$ spans an $I_{1}$-invariant subspace of $\operatorname{ind}_{I}^{G} \chi^{s}$ on which $I$ acts by $\chi^{r_{i} s}$. Moreover, the map $v^{r_{i} s} \mapsto t_{i} r_{i} v^{s}$ induces via Frobenius reciprocity isomorphisms $\nu_{r_{i} s}^{s}: \operatorname{ind}_{I}^{G} \chi^{r_{i} s} \rightarrow \operatorname{ind}_{I}^{G} \chi^{s}$.

Proof. First, since $\left(t_{i} r_{i}\right)^{-1} I_{1} t_{i} r_{i}$ is $I_{1}, t_{i} r_{i} v^{s}$ spans an $I_{1}$-invariant subspace. Second, we have that $t t_{i} r_{i} v^{s}=t_{i} r_{i}\left(r_{i}^{-1} t r_{i}\right) v^{s}=\chi^{r_{i} s}(t) t_{i} r_{i} v^{s}$ for any $t \in \mathbf{T}\left(\mathbf{Z}_{p}\right)$.

For the second statement, note that the composition

$$
\nu_{r_{i} s}^{s} \circ \nu_{s}^{r_{i} s}: \operatorname{ind}_{I}^{G} \chi^{s} \rightarrow \operatorname{ind}_{I}^{G} \chi^{r_{i} s} \rightarrow \operatorname{ind}_{I}^{G} \chi^{s},
$$

which maps $v^{s}$ to $r_{i} t_{i} r_{j} t_{j} v^{s}=\left(\begin{array}{lll}p & 0 & 0 \\ 0 & p & 0 \\ 0 & 0 & p\end{array}\right) v^{s}$, is invertible and hence an isomorphism.

Let $U_{i}$ be the operator on $\operatorname{ind}_{I}^{G} \chi^{s}$ given by $v^{s} \mapsto \sum_{g \in t_{i}^{-1} I_{1} t_{i} /\left(t_{i}^{-1} I_{1} t_{i} \cap I_{1}\right)} t_{i} g v^{s}$. 
Proposition 3.3.2. The composition

$$
\iota_{r_{i} s}^{s} \circ \nu_{s}^{r_{i} s}: \operatorname{ind}_{I}^{G} \chi^{s} \stackrel{\sim}{\rightarrow} \operatorname{ind}_{I}^{G} \chi^{r_{i} s} \hookrightarrow \operatorname{ind}_{I}^{G} \chi^{s}
$$

is the operator $U_{j}$.

Proof. From the respective formulas in 2.2.1 and Proposition 3.3.1, we see that the composition is defined by

$$
\begin{aligned}
v^{r_{i} s} \mapsto \sum_{g \in I_{1} /\left(r_{i} I_{1} r_{i}^{-1} \cap I_{1}\right)}\left(t_{j} r_{j}\right)\left(g r_{i}\right) v^{r_{i} s} & =\sum_{g \in r_{i}^{-1} I_{1} r_{i} /\left(r_{i}^{-1} I_{1} r_{i} \cap I_{1}\right)} t_{j} g v^{s} \\
& =\sum_{g \in t_{j}^{-1}} \sum_{I_{1} t_{j} /\left(t_{j}^{-1} I_{1} t_{j} \cap I_{1}\right)} t_{j} g v^{s} .
\end{aligned}
$$

\section{Patching}

Following [EGS15, we use Kisin's local-global modification of the Taylor-Wiles method to study lattices in completed cohomology in families over local deformation rings. In this section, we introduce the Taylor-Wiles patching method, as in $\mathrm{CEG}^{+} 16$, in order to deduce Theorem 4.1.5. This result crucially shows that patched modules have a natural action by integral Hecke algebras at $p$.

4.1. Galois representations and automorphic forms. We now consider some Galois representations and automorphic forms relevant to our implementation of the Taylor-Wiles patching method. Let $F$ be a CM field with $F^{+}$its totally real subfield such that $F / F^{+}$is unramified at all finite places and places of $F^{+}$dividing $p$ split in $F$.

4.1.1. Galois representations. Following [CHT08, §2], let $\mathcal{G} / \mathbf{Z}$ be the group scheme defined to be the semidirect product of $\mathrm{GL}_{3} \times \mathrm{GL}_{1}$ by the group $\{1, j\}$, which acts on $\mathrm{GL}_{3} \times \mathrm{GL}_{1}$ by $j(g, s)=\left(s \cdot\left(g^{-1}\right)^{t}, s\right)$. Let $E$ be a finite extension of $\mathbf{Q}_{p}$ with ring of integers $\mathcal{O}_{E}$, uniformizer $\varpi_{E}$, and residue field $k_{E}$. We consider a continuous Galois representation $\bar{\rho}: G_{F^{+}} \rightarrow \mathcal{G}\left(k_{E}\right)$ such that $\bar{r}^{-1}\left(\mathrm{GL}_{3}\left(k_{E}\right) \times \mathrm{GL}_{1}\left(k_{E}\right)\right)=G_{F}$. Then the composition $\bar{r}: G_{F} \rightarrow \mathrm{GL}_{3}\left(k_{E}\right) \times \mathrm{GL}_{1}\left(k_{E}\right) \rightarrow \mathrm{GL}_{3}\left(k_{E}\right)$ of $\left.\bar{\rho}\right|_{G_{F}}$ and the first projection is an essentially conjugate self-dual Galois representation. Recall that $\epsilon$ denotes the cyclotomic character and $\bar{\epsilon}$ its reduction. Assume that $\bar{r}^{c} \cong \bar{r}^{\vee} \bar{\epsilon}^{-2}$ where ${ }^{c}$ denotes complex conjugation. Suppose that $\bar{r}$ ramifies only at places that are split in $F / F^{+}$.

Axiom 4.1.1. We say that $\bar{r}$ satisfies the Taylor-Wiles conditions (TW) if

- $\bar{r}$ has image containing $\mathrm{GL}_{3}(\mathbf{F})$ for some $\mathbf{F} \subset k_{E}$ with $\# \mathbf{F}>9$, and

- $\bar{F}^{\text {ker ad } \bar{r}}$ does not contain $F\left(\zeta_{p}\right)$.

Note that the first condition, which is stronger than the usual condition of adequacy (see [Tho12, Definition 2.3]), allows us to choose a place $v_{1}$ of $F^{+}$which is prime to $p$ and satisfies the following properties (see $\left.\mathrm{CEG}^{+} 16, \S 2.3\right]$ ).

- $v_{1}$ splits in $F$ as $v_{1}=w_{1} w_{1}^{c}$

- $v_{1}$ does not split completely in $F\left(\zeta_{p}\right)$.

- $\bar{r}\left(\right.$ Frob $\left._{w_{1}}\right)$ has distinct $\mathbf{F}$-rational eigenvalues, no two of which have ratio $\left(\mathrm{N} v_{1}\right)^{ \pm 1}$. 
See Remark 4.1.2 for an explanation of the choice of $v_{1}$.

Let $\Sigma$ be a finite set of places of $F^{+}$away from $p$ such that $\bar{r}$ is unramified away from places dividing $p$ and those in $\Sigma$. Let $S$ be the set of places $v_{1}$, those in $\Sigma$, and those dividing $p$ in $F^{+}$. For each place $v$ in $S$, fix a place $\widetilde{v}$ in $F$ lying over $v$ and let $\widetilde{S}$ be the set of these places $\widetilde{v}$. For places $w$ of $F$, let $R_{w}^{\square}$ be the universal $\mathcal{O}_{E}$-lifting ring of $\left.\bar{r}\right|_{G_{F w}}$. For $v \in \Sigma$, let $R_{\widetilde{v}}^{\square, \tau_{\widetilde{v}}}$ be the reduced $p$-torsion free quotient of $R_{\widetilde{v}}^{\square}$ corresponding to lifts of the inertial type $\tau_{\widetilde{v}}$.

4.1.2. The ring $R_{\infty}$. Let $\delta_{F / F^{+}}$denote the quadratic character of $F / F^{+}$. Then we define

$$
\mathcal{S}=\left(F / F^{+}, S, \widetilde{S}, \mathcal{O}_{E}, \bar{r}, \epsilon^{-2} \delta_{F / F^{+}},\left\{R_{\widetilde{v}_{1}}^{\square}\right\}\left\{R_{\widetilde{v}}^{\square}\right\}_{v \mid p} \cup\left\{R_{\widetilde{v}}^{\square, \tau_{\widetilde{v}}}\right\}_{v \in \Sigma}\right)
$$

to be the deformation problem in the terminology of [CHT08. There is a universal deformation ring $R_{\mathcal{S}}^{\text {univ }}$ and a universal $S$-framed deformation ring $R_{\mathcal{S}}^{\square}$ in the sense of [CHT08, Definition 1.2.1].

Let

$$
R^{\text {loc }}=\widehat{\otimes}_{v \mid p} R_{\widetilde{v}}^{\square} \widehat{\otimes}\left(\widehat{\otimes}_{v \in \Sigma} R_{\widetilde{v}}^{\square, \tau_{\widetilde{v}}}\right) \widehat{\otimes} R_{\widetilde{v}_{1}}^{\square}
$$

where all completed tensor products are taken over $\mathcal{O}_{E}$. Choose an integer $q \geq$ $3\left[F^{+}: \mathbf{Q}\right]$ as in [Tho12, Proposition 4.4]. We introduce the ring

$$
R_{\infty}=R^{\text {loc }}\left[\left[x_{1}, \ldots, x_{q-3\left[F^{+}: \mathbf{Q}\right]}\right]\right],
$$

over which we construct a patched module of automorphic forms in 4.2 .

4.1.3. Automorphic forms on definite unitary groups. As in $\mathrm{CEG}^{+} 16$, $\left.\$ 2.3\right]$, we let $\mathbf{U} / \mathcal{O}_{F^{+}}$be a model for a definite unitary group that is quasisplit at all finite places of $F^{+}$and splits over $F$. For each place $v$ of $F^{+}$that splits as $v=w w^{c}$ in $F$, let $\mathcal{O}_{v}$ and $\mathcal{O}_{w}$ denote $\mathcal{O}_{F_{v}^{+}}$and $\mathcal{O}_{F_{w}}$, respectively. Let $\iota_{w}: \mathbf{U}\left(\mathcal{O}_{v}\right) \stackrel{\sim}{\rightarrow} \mathrm{GL}_{3}\left(\mathcal{O}_{w}\right)$ be the natural isomorphism. For $r$ as in $\$ 4.1 .1$ one defines a corresponding ideal $\lambda \subset \mathbf{T}$, where $\mathbf{T}=\mathbf{T}^{S_{p}, \text { univ }}$ is defined in $\left[\mathrm{CEG}^{+} 16, \S 2.3\right]$.

For a compact open subgroup $U \subset \mathbf{U}\left(\mathbf{A}_{F^{+}}^{\infty}\right)$ and a $\mathcal{O}_{E}[U]$-module $V$, we define the $\mathcal{O}_{E}$-module of algebraic modular forms $S(U, V)$ to be the space of functions

$$
f: \mathbf{U}\left(F^{+}\right) \backslash \mathbf{U}\left(\mathbf{A}_{F^{+}}^{\infty}\right) \rightarrow V
$$

such that $f(g u)=u^{-1} f(g)$ for all $g \in \mathbf{U}\left(\mathbf{A}_{F^{+}}^{\infty}\right)$ and $u \in U$.

For $m \in \mathbf{N}$, let $U_{m}=\prod_{v} U_{m, v} \subset \mathbf{U}\left(\mathbf{A}_{F^{+}}^{\infty}\right)$ be the compact open subgroup where

- $U_{m, v}=\mathbf{U}\left(\mathcal{O}_{v}\right)$ for all places $v$ which split in $F$ other than $v_{1}$ and those dividing $p$;

- $U_{m, v_{1}}$ is the preimage of the upper triangular matrices under the map

$$
\mathbf{U}\left(\mathcal{O}_{v_{1}}\right) \rightarrow \mathbf{U}\left(k_{v_{1}}\right) \underset{\iota_{\tilde{v}_{1}}}{\stackrel{\sim}{\rightarrow}} \mathrm{GL}_{3}\left(k_{\widetilde{v}_{1}}\right) ;
$$

- $U_{m, v}$ is the kernel of the map $\mathbf{U}\left(\mathcal{O}_{v}\right) \rightarrow \mathbf{U}\left(\mathcal{O}_{v} / p^{m}\right)$ for $v \mid p$;

- $U_{m, v}$ is a hyperspecial maximal compact open subgroup of $\mathbf{U}\left(F_{v}\right)$ if $v$ is inert in $F$.

Remark 4.1.2. Note that $v_{1}$ was chosen in 4.1 .1 so that for all $t \in \mathbf{U}\left(\mathbf{A}_{F^{+}}^{\infty}\right)$, $t^{-1} \mathbf{U}\left(F^{+}\right) t \cap U$ does not contain an element of order $p$, necessary for Theorem 4.1.5 (1). 
Let $\bar{r}, \Sigma, \widetilde{v}_{1}$, and $\tau_{\widetilde{v}}$ be as in $\$ 4.1 .1$. For $v \in \Sigma$, denote by $\sigma\left(\tau_{v}\right)$ the $\mathbf{U}\left(\mathcal{O}_{v}\right) \underset{\iota_{\tilde{v}}}{\stackrel{\sim}{\vec{v}}}$ $\mathrm{GL}_{3}\left(\mathcal{O}_{\widetilde{v}}\right)$-representation over $E$ which is the type corresponding to $\tau_{\widetilde{v}}$ by results towards inertial local Langlands (see [BC09, Proposition 6.5.3]). Note that $\sigma\left(\tau_{v}\right)$ does not depend on the choice of place $\widetilde{v} \mid v$ of $F$. For each $v \in \Sigma$, let $\sigma^{\circ}\left(\tau_{v}\right)$ be a $\mathbf{U}\left(\mathcal{O}_{v}\right)$-invariant $\mathcal{O}_{E}$-lattice in $\sigma\left(\tau_{v}\right)$, and $\bar{\sigma}\left(\tau_{v}\right)$ be its reduction. Let $\sigma^{\circ}(\tau)$ be $\otimes_{v \in \Sigma} \sigma^{\circ}\left(\tau_{v}\right)$ and $\bar{\sigma}(\tau)$ be $\otimes_{v \in \Sigma} \bar{\sigma}\left(\tau_{v}\right)$. Let $\widehat{H}^{0}$ and $\bar{H}^{0}$ be $\lim _{r} \lim _{m} S\left(U_{m}, \sigma^{\circ}(\tau) / \pi_{E}^{r}\right)$ and $\lim _{m} S\left(U_{m}, \bar{\sigma}(\tau)\right)$, respectively. These are spaces of algebraic modular forms with level at $\widetilde{v}_{1}$, infinite level at places dividing $p$, and coefficients at places in $\Sigma$.

Let $\mathbb{T}^{\Sigma \cup p \cup\left\{v_{1}\right\} \text {, univ }}$ be the commutative $\mathcal{O}_{E}$-algebra generated by the formal variables $T_{w}^{(j)}$ for $1 \leq j \leq 3$, where $w$ is a place of $F$ lying over a place $v$ of $F^{+}$away from $v_{1}, p$, and $\Sigma$ which splits as $w w^{c}$ in $F$. Then $\mathbb{T}^{\text {univ }}$ acts naturally on $\widehat{H}^{0}$ and $\bar{H}^{0}$ as in EG14, \$5.3]. For a Galois representation $r: G_{F} \rightarrow \mathrm{GL}_{3}\left(\mathcal{O}_{E}\right)$ lifting $\bar{r}$, let $\lambda$ be the ideal generated by the relations given by $\operatorname{det}\left(X I-r\left(\operatorname{Frob}_{w}\right)\right)=$ $X^{3}-(\mathbf{N} w) T_{w}^{(1)} X^{2}+(\mathbf{N} w) T_{w}^{(2)} X-(\mathbf{N} w) T_{w}^{(3)}$ for $w$ as above. Similarly define the ideal $\mathfrak{m} \subset \mathbb{T}^{\text {univ }}$ with respect to $\bar{r}$ so that $\mathfrak{m}=\lambda+\left(\varpi_{E}\right)$.

Axiom 4.1.3. We say that $r$ is modular (M) if

$$
\operatorname{Hom}_{\prod_{v \mid p} \mathbf{U}\left(\mathcal{O}_{v}\right)}\left(W, \widehat{H}^{0}\right)[\lambda] \neq 0
$$

for some finite locally algebraic $\mathcal{O}_{E}\left[\prod_{v \mid p} \mathbf{U}\left(\mathcal{O}_{v}\right)\right]$-module $W$.

We now assume that $r$ is modular. In particular, we have that $r^{c} \cong r^{\vee} \epsilon^{-2}, r$ is unramified outisde of $\Sigma$, and the inertial type of $\left.r\right|_{G_{F_{\widetilde{v}}}}$ is $\tau_{\widetilde{v}}$ for all places $v \in \Sigma$. Let $\pi$ and $\bar{\pi}$ be $\widehat{H}^{0}[\lambda]$ and $\bar{H}^{0}[\lambda]$.

Remark 4.1.4. As explained in the section following $\mathrm{CEG}^{+} 16$, Remark 2.9], one chooses a surjection $R_{\infty} \rightarrow R_{\mathcal{S}}^{\square}$.

With the choice in the previous remark fixed, let $\wp \subset \mathfrak{m} \subset R_{\infty}$ be the inverse images of the ideals in $R_{\mathcal{S}}^{\square_{S}}$ corresponding to $r$ and $\bar{r}$. Though an abuse of notation, the two uses of $\mathfrak{m}$ are compatible via the usual map from the deformation ring to the Hecke algebra. The following theorem summarizes some of the main results of $\mathrm{CEG}^{+} 16$. The proof is postponed to 4.2 .

Theorem 4.1.5. There exists an $R_{\infty} \llbracket \prod_{v \mid p} \mathbf{U}\left(\mathcal{O}_{v}\right) \rrbracket$-module $M_{\infty}$ with a compatible action of $\prod_{v \mid p} \mathbf{U}\left(F_{v}^{+}\right)$satisfying the following properties. For a $\prod_{v \mid p} \mathbf{U}\left(\mathcal{O}_{v}\right)$ representation $\otimes_{v \mid p} W_{v}$ which is finite over $\mathbf{Z}_{p}$, let $M_{\infty}\left(\otimes_{v \mid p} W_{v}\right)$ denote

$$
\operatorname{Hom}_{\prod_{v \mid p} \mathbf{U}\left(\mathcal{O}_{v}\right)}\left(\otimes_{v \mid p} W_{v}, M_{\infty}^{\vee}\right)^{\vee},
$$

where $\cdot \vee$ denotes the Pontrjagin dual. Then

(1) $M_{\infty}$ is projective as an $\mathcal{O}_{E} \llbracket \prod_{v \mid p} \mathbf{U}\left(\mathcal{O}_{v}\right) \rrbracket$-module;

(2) for $\otimes_{v \mid p} W_{v}$ as above which is either a p-power-torsion or p-torsion-free locally algebraic representation, $M_{\infty}\left(\otimes_{v \mid p} W_{v}\right)$ is maximal Cohen-Macaulay over its scheme-theoretic support in $\operatorname{Spec} R_{\infty}$ or is 0 ; and

(3) $\pi=M_{\infty}^{d}[\wp]$ and $\bar{\pi}=M_{\infty}^{\vee}[\mathfrak{m}]$, where ${ }^{d}$ denotes the Schikhof dual (see $\left[\mathrm{CEG}^{+} 16, \S 1.8\right]$ ).

Remark 4.1.6. By $\left[\mathrm{CEG}^{+} 16\right.$, Lemma 4.14], if $\otimes_{v} W_{v}$ is $\varpi_{E}$-torsion-free, then

$$
M_{\infty}\left(\otimes_{v} W_{v}\right) \cong \operatorname{Hom}_{K}\left(\otimes_{v} W_{v}, M_{\infty}^{d}\right)^{d} .
$$


Note that in $\mathrm{CEG}^{+} 16, M_{\infty}\left(\otimes_{v} W_{v}\right)$ is only defined for $p$-torsion-free objects and the definition with Schikhof duality is used instead.

4.2. The construction of patched modules. In this section, we describe the construction of the patched module $M_{\infty}$ satisfying the properties in Theorem 4.1.5. The main difference from $\mathrm{CEG}^{+} 16$ is that we allow ramification away from $p$, and we patch at all places dividing $p$ simultaneously. The necessary modifications are straightforward and the proofs are identical.

4.2.1. Compact open subgroups and Taylor-Wiles primes. Recall the definition of $U_{m, v}$ for $m \in \mathbf{N}$ and $v$ a finite place of $F^{+}$from $\$ 4.1 .3$. We now choose TaylorWiles primes. Recall that in 4.1 .1 we chose an integer $q$. As in $\left[\mathrm{CEG}^{+} 16, \S 2.6\right]$ (which follows [Tho12, §4]), for each $N \geq 1$ we use (TW) to choose a set $Q_{N}$ of $q$ places of $F^{+}$satisfying the following properties: for each place $v \in Q_{N}$,

- $v$ is not in $\Sigma$, does not divide $p$, and splits in $F$;

- $\mathrm{N} v \equiv 1 \bmod p^{N}$

- if $w \mid v$ is a place of $F$, then $\left.\bar{r}\right|_{G_{F_{w}}}$ has a nonzero direct summand $\bar{\psi}_{w}$ which is an eigenspace on which Frobenius acts semisimply.

For each $v \in Q_{N}$, choose a place $\widetilde{v} \mid v$ of $F$ and let $\widetilde{Q}_{N}=\left\{\widetilde{v}: v \in Q_{N}\right\}$. For $v \in Q_{N}$, we let $U_{1}\left(Q_{N}\right)_{v} \subset \mathbf{U}\left(\mathcal{O}_{v}\right)$ be the corresponding parahoric compact open subgroup defined in [EG14, §5.5]. Let $U_{1}\left(Q_{N}\right)_{m, v}=U_{m, v}$ for $v \notin Q_{N}$ and $U_{1}\left(Q_{N}\right)_{m, v}=U_{1}\left(Q_{N}\right)_{v}$ for $v \in Q_{N}$. We define the compact open subgroup $U_{1}\left(Q_{N}\right)_{m}=\prod_{v} U_{1}\left(Q_{N}\right)_{m, v} \subset \mathbf{U}\left(\mathbf{A}_{F^{+}}^{\infty}\right)$. Recall from $\$ 4.1 .3$ the definition of the set of places $\Sigma$ and $\sigma^{\circ}(\tau)$.

For each $v \in Q_{N}$, let $R_{\widetilde{v}}^{\bar{\psi}_{\tilde{v}}}$ be the quotient of $R_{\widetilde{v}}^{\square}$ defined in [EG14, $\left.\S 5.5\right]$. We let $\mathcal{S}_{Q_{N}}$ be the deformation problem

$$
\begin{aligned}
\mathcal{S}_{Q_{N}}= & \left(F / F^{+}, S \cup Q_{N}, \widetilde{S} \cup \widetilde{Q}_{N}, \mathcal{O}_{E}, \bar{r}, \epsilon^{-2} \delta_{F / F^{+}},\right. \\
& \left.\left\{R_{\widetilde{v}_{1}}^{\square}\right\} \cup\left\{R_{\widetilde{v}}^{\square}\right\}_{v \mid p} \cup\left\{R_{\widetilde{v}}^{\square, \tau_{\widetilde{v}}}\right\}_{v \in \Sigma} \cup\left\{R_{\widetilde{v}}^{\bar{\psi}_{\widetilde{v}}}\right\}_{v \in Q_{n}}\right) .
\end{aligned}
$$

Again, we have the universal deformation ring $R_{\mathcal{S}_{Q_{N}}}^{\text {univ }}$ and the universal $S$-framed deformation ring $R_{\mathcal{S}_{Q_{N}}}^{\square}$. The above properties of the set $Q_{N}$ crucially guarantee that $R_{\mathcal{S}_{Q_{N}}}^{\square}$ is topologically generated over $R^{\text {loc }}$ by $q-\left[F^{+}: \mathbf{Q}\right] n(n-1) / 2$ elements. Let $M_{1, Q_{N}}=\operatorname{pr}\left(\left(S\left(U_{1}\left(Q_{N}\right)_{2 N}, \sigma^{\circ}(\tau) / \varpi^{N}\right)_{\mathfrak{m}_{Q_{N}}}\right)^{\vee}\right.$ where $\cdot{ }^{\vee}$ denotes the Pontrjagin dual, pr is defined in $\left[\mathrm{CEG}^{+} 16, \S 2.6\right]$, and $\mathfrak{m}_{Q_{N}} \subset \mathbb{T}^{\Sigma \cup p \cup\left\{v_{1}\right\} \cup Q_{N} \text {, univ }}$ is the maximal ideal defined as in 4.1 .3 . Let

$$
M_{1, Q_{N}}^{\square}=M_{1, Q_{N}} \otimes_{R_{\mathcal{S}_{Q_{N}}}^{\text {univ }}} \quad R_{\mathcal{S}_{Q_{N}}}^{\square_{S}}
$$

and

$$
S_{\infty}=\mathcal{O}_{E}\left[\left[z_{1}, \ldots, z_{9 \# S}, y_{1}, \ldots, y_{q}\right]\right] .
$$

As in $\left[\mathrm{CEG}^{+} 16, \S 2.8\right]$, we patch the spaces $M_{1, Q_{N}}^{\square}$ together using the Taylor-Wiles method to get an $R_{\infty}\left[\left[\prod_{v \mid p} \mathbf{U}\left(\mathcal{O}_{v}\right)\right]\right]$-module $M_{\infty}$.

Proof of Theorem 4.1.5. For part (1) of Theorem 4.1.5 the proof of $\mathrm{CEG}^{+} 16$, Proposition 2.10] shows that $M_{\infty}$ is in fact projective as an $S_{\infty}\left[\left[\prod_{v \mid p} \mathbf{U}\left(\mathcal{O}_{v}\right)\right]\right]-$ module and admits a $\prod_{v \mid p} \mathbf{U}\left(F_{v}^{+}\right)$-action that extends the action of $\prod_{v \mid p} \mathbf{U}\left(\mathcal{O}_{v}\right)$. 
Part (2) follows from [CEG ${ }^{+}$16, Lemma 4.18(1)]. Part (3) follows directly from the construction of $M_{\infty}$ (see $\left.\left[\mathrm{CEG}^{+} 16, \S 2.8\right]\right)$.

\section{Cyclicity of Patched modules}

We deduce axiomatic mod $p$ multiplicity one results (see Theorems 5.3 .2 and 5.3.5 for upper alcove algebraic vectors and tame principal series types. Let $F / F^{+}$ and $r$ and $\bar{r}$ be as in 4 . Throughout $\$ 5$, assume that $p$ splits completely in $F$. For each place $v \mid p$ of $F^{+}$, choose a place $\widetilde{v} \mid v$ of $F$. For $\mathbf{U}\left(\mathcal{O}_{v}\right) \underset{\iota_{\tilde{v}}}{\stackrel{\sim}{\rightarrow}} \mathrm{GL}_{3}\left(\mathcal{O}_{\widetilde{v}}\right)-$ representations $V_{\widetilde{v}}$, let $M_{\infty}\left(\otimes_{v} V_{\widetilde{v}}\right)$ be defined as in Theorem 4.1.5. Fix a place $v \mid p$ of $F^{+}$. In what follows, we will sometimes fix a choice of $\mathrm{GL}_{3}\left(\mathcal{O}_{\widetilde{v}^{\prime}}\right)$-representation $V_{\widetilde{v}^{\prime}}$ for each $v^{\prime} \neq v$ and simply write $M_{\infty}\left(V_{\widetilde{v}}\right)$ for a $\mathrm{GL}_{3}\left(\mathcal{O}_{\widetilde{v}}\right)$-representation $V_{\widetilde{v}}$ to denote $M_{\infty}\left(V_{\widetilde{v}} \otimes_{v^{\prime} \neq v} V_{\widetilde{v}^{\prime}}\right)$.

5.1. A key isomorphism. In this section, we prove Lemma 5.1.5 which provides the key input in proving cyclicity of patched modules for upper alcove algebraic representations. Suppose that $\bar{r}$ satisfies (TW) and $r$ satisfies (M) from $\$ 4.1 .1$ and 4.1 .3 Fix a place $v \mid p$ of $F^{+}$. We say that $\bar{r}$ is modular of $\mathrm{GL}_{3}\left(\mathcal{O}_{\widetilde{v}}\right)$-weight $\sigma$ at $\widetilde{v}$ if $\operatorname{Hom}_{\mathrm{GL}_{3}\left(\mathcal{O}_{\tilde{v}}\right)}(\sigma, \bar{\pi})$ is nonzero. Note that since we define $\mathfrak{m}$ in terms of $\bar{r}$ rather than $\bar{r}^{\vee}$ and use $\operatorname{Hom}_{\mathrm{GL}_{3}\left(\mathcal{O}_{\tilde{v}}\right)}(\sigma, \bar{\pi})$ rather than $(\sigma \otimes \bar{\pi})^{\mathrm{GL}_{3}\left(\mathcal{O}_{\tilde{v}}\right)}$, our definition agrees with that of [Her09, EGH13]. We denote the set of modular Serre weights for $\bar{r}$ at $\widetilde{v}$ by $W_{\widetilde{v}}(\bar{r})$. Note that the corresponding set of $\mathbf{U}\left(\mathcal{O}_{v}\right)$-representations does not depend on the choice of $\widetilde{v}$. The following is an immediate corollary of Theorem 4.1.5.3.

Proposition 5.1.1. A weight $\sigma$ belongs to $W_{\widetilde{v}}(\bar{r})$ if and only if $M_{\infty}(\sigma) \neq 0$ for some choice of $V_{\widetilde{v}^{\prime}}$.

We introduce the following axiom.

Axiom 5.1.2. We say that $\bar{r}$ satisfies weight elimination (WE) for $\mu$ at $\widetilde{v}$ if $\operatorname{Ind}_{\widetilde{P}_{\mu}}^{K} \sigma_{N_{\mu}}^{\prime} / \bar{W}$ has no Jordan-Hölder factors in $W_{\widetilde{v}}(\bar{r})$.

Example 5.1.3. Suppose that $\left.r\right|_{F_{\tilde{v}}}$ is irreducible with $\left.\bar{r}\right|_{I_{\tilde{v}}} \cong \psi \oplus \psi^{p} \oplus \psi^{p^{2}}$ where $\psi \cong \omega_{3}^{-\left(a_{\tilde{v}}+1\right)-p\left(b_{\tilde{v}}+1\right)-p^{2}\left(c_{\tilde{v}}+1\right)}$. Then, $\bar{r}$ satisfies (WE) for $\mu_{1}$ at $\widetilde{v}$ by EGH13, Theorem 7.5.5] (see Her06, Theorem 5.1 and Conjecture 6.6] for a formula for $\left.W^{?}(\bar{r})\right)$.

Remark 5.1.4. By Proposition 5.1.1 and Theorem 4.1.5 1), if $\bar{r}$ satisfies (WE) for $\mu$ at $\widetilde{v}$ then $M_{\infty}\left(\operatorname{Ind}_{\widetilde{P}_{\mu}}^{K} \sigma_{N_{\mu}}^{\prime} / \bar{W}\right)=0$ and $M_{\infty}\left(\operatorname{ker}\left(\operatorname{Ind}_{\widetilde{P}_{-\mu}}^{K} \sigma^{\prime N_{-\mu}} \rightarrow \bar{W}^{0}\right)\right)=0$ for all choices of $V_{v^{\prime}}$.

An element of $\mathcal{H}\left(\bar{W}, \bar{W}^{0}\right)$ induces an $R_{\infty}$-homomorphism

$\operatorname{Hom}_{G \times \prod_{v^{\prime} \neq v} \mathbf{U}\left(\mathcal{O}_{v^{\prime}}\right)}\left(\operatorname{ind}_{K}^{G} \bar{W}^{0} \otimes \otimes_{v^{\prime} \neq v} V_{\widetilde{v}^{\prime}}, M_{\infty}^{\vee}\right) \rightarrow \operatorname{Hom}_{G \times \prod_{v^{\prime} \neq v} \mathbf{U}\left(\mathcal{O}_{v^{\prime}}\right)}\left(\operatorname{ind}_{K}^{G} \bar{W} \otimes \otimes_{v^{\prime} \neq v} V_{\widetilde{v}^{\prime}}, M_{\infty}^{\vee}\right)$,

and by duality an element of $\operatorname{Hom}_{R_{\infty}}\left(M_{\infty}(\bar{W}), M_{\infty}\left(\bar{W}^{0}\right)\right)$. This defines a ring homomorphism $\mathcal{H}\left(\bar{W}, \bar{W}^{0}\right) \rightarrow \operatorname{Hom}_{R_{\infty}}\left(M_{\infty}(\bar{W}), M_{\infty}\left(\bar{W}^{0}\right)\right)$. It is here that we use that the $R_{\infty} \llbracket \prod_{v^{\prime} \mid p} \mathbf{U}\left(\mathcal{O}_{v^{\prime}}\right) \rrbracket$-module $M_{\infty}$ in Theorem 4.1.5 has a compatible $\prod_{v^{\prime} \mid p} \mathbf{U}\left(F_{v^{\prime}}^{+}\right)$-action. By abuse of notation, we also denote the image of $T^{\prime}$ in $\operatorname{Hom}_{R_{\infty}}\left(M_{\infty}\left(W_{1}\right), M_{\infty}\left(W_{2}\right)\right)$ by $T^{\prime}$. 
Lemma 5.1.5. Suppose that $\bar{r}$ satisfies (WE) for $\mu$ at $\widetilde{v}$. Then the $R_{\infty}$-module homomorphism $T^{\prime}: M_{\infty}(\bar{W}) \rightarrow M_{\infty}\left(\bar{W}^{0}\right)$ is an isomorphism.

Proof. By Proposition 3.1 .4 and Theorem 4.1.5 the map $T^{\prime}: M_{\infty}(\bar{W}) \rightarrow M_{\infty}\left(\bar{W}^{0}\right)$ can be written as the composition

$$
M_{\infty}(\bar{W}) \rightarrow M_{\infty}\left(\operatorname{Ind}_{\widetilde{P}_{\mu}}^{K} \sigma_{N_{\mu}}\right) \rightarrow M_{\infty}\left(\operatorname{Ind}_{\widetilde{P}_{-\mu}}^{K} \sigma^{N_{-\mu}}\right) \rightarrow M_{\infty}\left(\bar{W}^{0}\right)
$$

By Frobenius reciprocity and Proposition 3.1 .3 , the second morphism is an isomorphism. By the exactness of $M_{\infty}(\cdot)$ from Theorem 4.1.5 1) and Remark 5.1.4, the first and third morphisms are isomorphisms.

5.2. Multiplicity one for extensions. From Lemma 5.1.5, we deduce cyclicity of certain patched modules, extending the method of Dia97] and [Fuj06]. Recall that $\tau_{\widetilde{v}}$ is the inertial type of $r$ at $\widetilde{v}$ for each place $v \in \Sigma$.

Axiom 5.2.1. We say that $r$ has regular type $(\mathbf{R T})$ if $\widehat{\otimes}_{v \in \Sigma} R_{\widetilde{v}}^{\square, \tau_{\tilde{v}}}$ is a regular local ring.

Remark 5.2.2. We note that the axiom (RT) is satisfied if $r$ is minimally ramified in the sense of [CHT08, Definition 2.4.14] by [CHT08, Corollary 2.4.21].

Suppose that for each place $v \mid p$ of $F, \sigma_{\widetilde{v}}$ is a Serre weight $F\left(x_{\widetilde{v}}, y_{\widetilde{v}}, z_{\widetilde{v}}\right)$ with $x_{\widetilde{v}} \geq y_{\widetilde{v}} \geq z_{\widetilde{v}}$ and $x_{\widetilde{v}}-z_{\widetilde{v}}<p-3$. The following theorem is a well-known consequence of the method of Diamond and Fujiwara.

Theorem 5.2.3. Assume that $r$ satisfies (RT). Then $M_{\infty}\left(\otimes_{v} \sigma_{\widetilde{v}}\right)$ is cyclic over $R_{\infty}$ or 0 .

Proof. Let $R_{\infty}\left(\otimes_{v} W\left(x_{\widetilde{v}}, y_{\widetilde{v}}, z_{\widetilde{v}}\right)\right)^{\prime}$ be the quotient

$$
\widehat{\otimes}_{v \mid p} R_{\widetilde{v}}^{\psi_{\widetilde{v}}, \square} \widehat{\otimes}\left(\widehat{\otimes}_{v \in \Sigma} R_{\widetilde{v}}^{\square, \tau_{\tilde{v}}}\right) \widehat{\otimes} R_{\widetilde{v}_{1}}^{\square}\left[\left[x_{1}, \ldots, x_{q-3\left[F^{+}: \mathbf{Q}\right]}\right]\right]
$$

of $R_{\infty}$, where $R_{\widetilde{v}}^{\psi_{\tilde{v}}, \square}$ is the crystalline lifting ring of $\left.\bar{r}\right|_{G_{F_{\tilde{v}}}}$ of Hodge-Tate weights $\left(x_{\widetilde{v}}+2, y_{\widetilde{v}}+1, z_{\widetilde{v}}\right)$. Since the Hodge-Tate weights $\left(x_{\widetilde{v}}+2, y_{\widetilde{v}}+1, z_{\widetilde{v}}\right)$ are in the Fontaine-Laffaille range, $R_{\widetilde{v}}^{\psi_{\widetilde{v}}, \square}$ is formally smooth over $\mathcal{O}$ by [CHT08, Lemmas 2.4.27 and 2.4.28]. By the choice of $v_{1}, R_{\widetilde{v}_{1}}^{\square}$ is formally smooth over $\mathcal{O}$ by [CHT08, Lemma 2.5]. Finally, $R_{\widetilde{v}}^{\square, \tau_{\tilde{v}}}$ is formally smooth over $\mathcal{O}$ for all $v \in \Sigma$ by (RT). We conclude that $R_{\infty}\left(\otimes_{v} W\left(x_{\widetilde{v}}, y_{\widetilde{v}}, z_{\widetilde{v}}\right)\right)^{\prime}$ is formally smooth over $\mathcal{O}$.

Assume that $M_{\infty}\left(\otimes_{v} W\left(x_{\widetilde{v}}, y_{\widetilde{v}}, z_{\widetilde{v}}\right)\right)$ is nonzero. Then by the proof of $\left[\mathrm{CEG}^{+} 16\right.$, Lemma 4.18], it is maximal Cohen-Macaulay over $R_{\infty}\left(\otimes_{v} W\left(x_{\widetilde{v}}, y_{\widetilde{v}}, z_{\widetilde{v}}\right)\right)^{\prime}$. By Dia97, Theorem 2.1], $M_{\infty}\left(\otimes_{v} W\left(x_{\widetilde{v}}, y_{\widetilde{v}}, z_{\widetilde{v}}\right)\right)$ is a free module over $R_{\infty}\left(\otimes_{v} W\left(x_{\widetilde{v}}, y_{\widetilde{v}}, z_{\widetilde{v}}\right)\right)^{\prime}$. By the proof of $\left[\mathrm{CEG}^{+} 16\right.$, Lemma $\left.4.18(1)\right], M_{\infty}\left(\otimes_{v} W\left(x_{\widetilde{v}}, y_{\widetilde{v}}, z_{\widetilde{v}}\right)\right)[1 / p]$ is locally free of rank at most one over $R_{\infty}\left(\otimes_{v} W\left(x_{\widetilde{v}}, y_{\widetilde{v}}, z_{\widetilde{v}}\right)\right)^{\prime}[1 / p]$. Thus $M_{\infty}\left(\otimes_{v} W\left(x_{\widetilde{v}}, y_{\widetilde{v}}, z_{\widetilde{v}}\right)\right)$, and therefore its reduction $M_{\infty}\left(\otimes_{v} \sigma_{\widetilde{v}}\right)$, is free of rank one over its support.

We fix a place $v \mid p$ of $F^{+}$and continue to use the notation of 5.1 . Suppose that $V_{\widetilde{v}^{\prime}}$ is $p$-torsion free for all $v^{\prime} \neq v$.

Lemma 5.2.4. Suppose that $\bar{r}$ satisfies (WE) for $\mu$ at $\widetilde{v}$ for some minuscule coweight $\mu$. Let $R^{\prime} \subset \operatorname{End}_{R_{\infty}}\left(M_{\infty}\left(W^{0}\right)\right)$ be a commutative local $R_{\infty}$-algebra which commutes with the action of $T$, and let $R$ be $R^{\prime}[T]$. If $M_{\infty}(\sigma)$ is nonzero, then $R$ is a local ring. 
Proof. By the definition of $T$ in 3.2 we have the commutative diagram

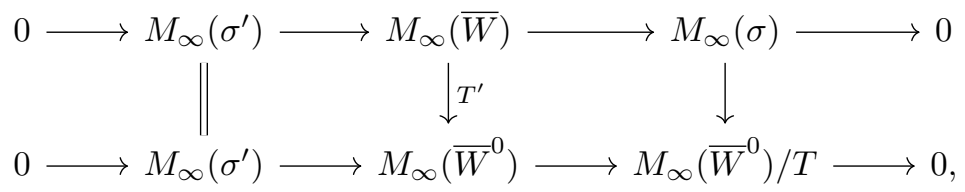

where the map $M_{\infty}\left(\sigma^{\prime}\right) \rightarrow M_{\infty}\left(\bar{W}^{0}\right)$ is defined to be the composition of the map $M_{\infty}\left(\sigma^{\prime}\right) \rightarrow M_{\infty}(\bar{W})$ and $T^{\prime}$. The middle vertical arrow is an isomorphism by Lemma 5.1.5 and hence so is the right vertical arrow. The module $M_{\infty}(\sigma)$ is cyclic and nonzero by assumption. Let $\mathfrak{a}=\left(z_{1}, \ldots, z_{9 \# S}, y_{1}, \ldots, y_{q}\right)$ be the augmentation ideal of $S_{\infty}$ in the notation of $\$ 4.2$. Then $M_{\infty}\left(W^{0}\right) / \mathfrak{a}$ is $p$-torsion free by $\mathrm{CEG}^{+}$16, Corollary 2.11]. By definition of $T$ and [BLGGT12, Theorem 1.2], $T$ acts on $\left(M_{\infty}\left(W^{0}\right) / \mathfrak{a}\right) \otimes_{\mathcal{O}_{E}} E$ semisimply. Since $M_{\infty}\left(W^{0}\right) /(\mathfrak{a}, T)$ is nonzero, each eigenvalue of the action of $T$ on $M_{\infty}\left(W^{0}\right) / \mathfrak{a} \otimes_{\mathcal{O}_{E}} E$ must have positive valuation. We conclude that the image of $T$ in $R / \mathfrak{a}$ must be in the Jacobson radical. Since $R$ is finite over $R_{\infty}$ (because $\operatorname{End}_{R_{\infty}}\left(M_{\infty}\right)$ is), any maximal ideal of $R$ must contain a. Thus, $T$ is in the Jacobson radical of $R$, and $R$ is local.

5.3. Multiplicity one for locally algebraic types. In this section, we prove some mod $p$ multiplicity one results for upper alcove algebraic vectors and principal series types. For $v^{\prime} \mid p$, let $a_{\widetilde{v}^{\prime}}, b_{\widetilde{v}^{\prime}}$, and $c_{\widetilde{v}^{\prime}}$ be integers such that $a_{\widetilde{v}^{\prime}}>b_{\widetilde{v}^{\prime}}>c_{\widetilde{v}^{\prime}}$ and $a_{\widetilde{v}^{\prime}}-c_{\widetilde{v}^{\prime}}<p-1$. Let $\sigma_{\widetilde{v}^{\prime}}=F\left(a_{\widetilde{v}^{\prime}}-1, b_{\widetilde{v}^{\prime}}, c_{\widetilde{v}^{\prime}}+1\right)$ and $\sigma_{\widetilde{v}^{\prime}}^{\prime}=F\left(c_{\widetilde{v}^{\prime}}+p-1, b_{\widetilde{v}^{\prime}}, a_{\widetilde{v}^{\prime}}-\right.$ $p+1)$.

Axiom 5.3.1. We say that $\bar{r}$ is modular of weight $\otimes_{v^{\prime}} \sigma_{\widetilde{v}^{\prime}}$ (M') if

$$
\operatorname{Hom}_{\prod_{v^{\prime} \mid p} \mathbf{U}\left(\mathcal{O}_{v^{\prime}}\right)}\left(\otimes_{v^{\prime}} \sigma_{\widetilde{v}^{\prime}}, \bar{\pi}\right) \neq 0 .
$$

By Theorem 4.1.5 3), (M') holds if and only if $M_{\infty}\left(\otimes_{v^{\prime}} \sigma_{\widetilde{v}^{\prime}}\right) \neq 0$.

5.3.1. The case of algebraic vectors. We continue to denote by $v$ a fixed place of $F^{+}$ dividing $p$ and continue to use the notation of 5.1 . Define $W_{\widetilde{v}^{\prime}}, W_{\widetilde{v}^{\prime}}^{0}, T_{\widetilde{v}^{\prime}}$, etc. as in 2.1 . Let $R^{\text {alg }} \subset \operatorname{End}_{R_{\infty}}\left(M_{\infty}\left(\otimes_{v^{\prime}} W_{\widetilde{v}^{\prime}}^{0}\right)\right)$ be the subring generated by the images of $R_{\infty}$ and $T_{\widetilde{v}^{\prime}}$.

Theorem 5.3.2. For each place $v^{\prime} \mid p$, let $\mu_{\widetilde{v}^{\prime}}$ be some minuscule coweight. Suppose that $\bar{r}$ satisfies $\left(\mathbf{M}^{\prime}\right),(\mathbf{R T})$, and $(\mathbf{W E})$ for $\mu_{\widetilde{v}^{\prime}}$ at $\widetilde{v}^{\prime}$. Then $R^{\text {alg }}$ is a local ring and $M_{\infty}\left(\otimes_{v^{\prime}} W_{\widetilde{v}^{\prime}}^{0}\right)$ is free of rank one over $R^{\text {alg. }}$.

Proof. By changing $R^{\prime}$ and $R$ as necessary, Lemma 5.2 .4 inductively shows that $R^{\text {alg }}$ is a local ring. Moreover, by the proof of Lemma 5.2.4 $M_{\infty}\left(\otimes_{v^{\prime}} W_{\widetilde{v}^{\prime}}^{0} /\left(\varpi_{E}, T_{\widetilde{v}^{\prime}}\right) \widetilde{v}^{\prime}\right)$ is isomorphic to $M_{\infty}\left(\otimes_{v^{\prime}} \sigma_{\widetilde{v}^{\prime}}\right)$. By Nakayama's lemma and Theorem 5.2.3. $M_{\infty}\left(\otimes_{v^{\prime}} W_{\widetilde{v}^{\prime}}^{0}\right)$ is a cyclic $R^{\text {alg }}$-module. By definition, it is also a faithful $R^{\text {alg }}$-module, and is thus free of rank one.

5.3.2. The case of principal series types. For places $v^{\prime} \mid p$ of $F^{+}$, let $\tau_{\widetilde{v}^{\prime}}=\operatorname{ind}_{I}^{K} \chi_{\widetilde{v}^{\prime}}$, where $\chi_{\widetilde{v}^{\prime}}=\eta^{a_{\widetilde{v}^{\prime}}} \otimes \eta^{b_{\widetilde{v}^{\prime}}} \otimes \eta^{c_{\widetilde{v}^{\prime}}}$. So $\tau_{\widetilde{v}^{\prime}}$ is a tame principal series $\mathrm{GL}_{3}\left(\mathcal{O}_{\widetilde{v}^{\prime}}\right)$-type.

Axiom 5.3.3. We say that $\bar{r}$ satisfies weight elimination (WE) for $\tau_{\widetilde{v}}$ and $\widetilde{v}$ if

$$
\begin{aligned}
W_{\widetilde{v}}(\bar{r}) \cap \operatorname{JH}\left(\bar{\tau}_{\widetilde{v}}\right) \subset\{ & F\left(a_{\widetilde{v}}-1, b_{\widetilde{v}}, c_{\widetilde{v}}+1\right), F\left(b_{\widetilde{v}}, c_{\widetilde{v}}, a_{\widetilde{v}}-p+1\right), \\
& \left.F\left(b_{\widetilde{v}}+p-1, a_{\widetilde{v}}, c_{\widetilde{v}}\right), F\left(c_{\widetilde{v}}+p-1, b_{\widetilde{v}}, a_{\widetilde{v}}-p+1\right)\right\} .
\end{aligned}
$$


Lemma 5.3.4. Suppose that $\bar{r}$ satisfies (M'), (RT), and (WE) for $\bar{\tau}_{\widetilde{v}}$ and $\widetilde{v}$ for some $v \mid p$. Let $R^{\prime} \subset \operatorname{End}_{R_{\infty}}\left(M_{\infty}\left(\tau^{s_{2}}\right)\right)$ be a commutative local $R_{\infty}$-algebra which commutes with the actions of $U_{1}$ and $U_{2}$, and let $R$ be $R^{\prime}\left[U_{1}, U_{2}\right]$. If $M_{\infty}(\sigma)$ is nonzero, then $R$ is a local ring.

Proof. The proof is similar to Lemma 5.2.4. By Proposition 3.3.2, we have isomorphisms

$$
M_{\infty}\left(\tau_{\widetilde{v}}^{s_{2}} / \iota_{s_{1}}^{s_{2}}\left(\tau_{\widetilde{v}}^{s_{1}}\right)\right) \stackrel{\sim}{\rightarrow} M_{\infty}\left(\tau_{\widetilde{v}}^{s_{2}}\right) / U_{2, \widetilde{v}}
$$

and

$$
M_{\infty}\left(\tau_{\widetilde{v}}^{s_{2}} / \iota_{w_{0}}^{s_{2}}\left(\tau_{\widetilde{v}}^{w_{0}}\right)\right) \stackrel{\sim}{\rightarrow} M_{\infty}\left(\tau_{\widetilde{v}}^{s_{2}}\right) / U_{1, \widetilde{v}}
$$

The left sides are nonzero since $M_{\infty}\left(\sigma_{\widetilde{v}}\right)$ is. Replacing $T$ in the proof of Lemma 5.2 .4 by $U_{1}$ and $U_{2}$, we see that $R$ is local.

Let $R^{\mathrm{ps}} \subset \operatorname{End}_{R_{\infty}}\left(M_{\infty}\left(\otimes_{v^{\prime} \mid p} \tau_{\widetilde{v}^{\prime}}^{s_{2}}\right)\right)$ denote the subring generated by the image of $R_{\infty}$ and the Hecke operators $U_{2, \widetilde{v}^{\prime}}$ and $U_{1, \widetilde{v}^{\prime}}$ for all $v^{\prime} \mid p$.

Theorem 5.3.5. Assume that $\bar{r}$ satisfies (M'), (RT), and (WE) for $\bar{\tau}_{\widetilde{v}^{\prime}}$ and $\widetilde{v}^{\prime}$ for all $v^{\prime} \mid p$. Then $R^{\mathrm{ps}}$ is a local ring and the $R^{\mathrm{ps}}{ }_{-}$module $M_{\infty}\left(\otimes_{v^{\prime} \mid p} \tau_{\widetilde{v}^{\prime}}^{s_{2}}\right)$ is free of rank one.

Proof. By changing $R^{\prime}$ and $R$ as necessary, Lemma 5.3.4 inductively shows that $R^{\mathrm{ps}}$ is a local ring. By (WE) for $\tau_{\widetilde{v}^{\prime}}$ and $\widetilde{v}^{\prime}$ for all $v^{\prime} \mid p$ and Proposition 2.2.2, the natural inclusion

$M_{\infty}\left(\otimes_{v^{\prime} \mid p} \sigma_{\widetilde{v}^{\prime}}\right) \rightarrow M_{\infty}\left(\otimes_{v^{\prime} \mid p} \bar{\tau}_{\widetilde{v}^{\prime}}^{s_{2}} /\left(\iota_{s_{1}}^{s_{2}}\left(\bar{\tau}_{\widetilde{v}^{\prime}}^{s_{1}}\right)+\iota_{w_{0}}^{s_{2}}\left(\bar{\tau}_{\widetilde{v}^{\prime}}^{w_{0}}\right)\right)\right) \cong M_{\infty}\left(\otimes_{v^{\prime} \mid p} \bar{\tau}_{\widetilde{v}^{\prime}}^{s_{2}}\right) /\left(U_{1, \widetilde{v}^{\prime}}, U_{2, \widetilde{v}^{\prime}}\right)_{v^{\prime} \mid p}$

is an isomorphism. By Nakayama's lemma and Theorem 5.2.3, $M_{\infty}\left(\otimes_{v^{\prime} \mid p} \tau_{\widetilde{v}^{\prime}}^{s_{2}}\right)$ is a cyclic $R^{\mathrm{ps}}$-module. Since it is also a faithful $R^{\mathrm{ps}}$-module, it is free of rank one.

5.3.3. An application of cyclicity. In this subsection, we deduce a lemma which allows one to use multiplicity one to relate the reduction of lattices to modularity of Serre weights. We again fix a place $v \mid p$ of $F^{+}$and continue to use the notation of $\$ 5.1$

Lemma 5.3.6. Fix a place $v \mid p$ and let $V_{\widetilde{v}^{\prime}}$ be $\mathcal{O}_{E}\left[\mathrm{GL}_{3}\left(\mathcal{O}_{\widetilde{v}}\right)\right]$-modules for $v^{\prime} \neq v$. Let $\tau$ be a finite locally algebraic $E\left[\mathrm{GL}_{3}\left(\mathcal{O}_{\tilde{v}}\right)\right]$-module, and let $\tau_{1} \subset \tau$ be an $\mathcal{O}_{E}$ lattice with irreducible cosocle $\sigma$ where $M_{\infty}(\sigma) \neq 0$. Let $R$ be a local $R_{\infty}$-algebra which acts on $M_{\infty}\left(\tau_{1}\right)$ extending the action of $R_{\infty}$. Suppose that $M_{\infty}\left(\tau_{1}\right)$ is a cyclic $R$-module and $\tau_{2} \subsetneq \tau_{1}$ is a sublattice. Then $M_{\infty}\left(\tau_{2}\right) \subset \mathfrak{m}_{R} M_{\infty}\left(\tau_{1}\right)$.

Proof. The inclusion $\tau_{2} \hookrightarrow \tau_{1}$ and surjection $\tau_{1} \rightarrow \sigma$, whose composition is 0 , induce the diagram

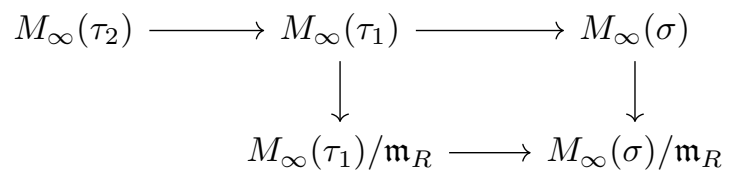

where the composition of the top row is 0. The map $M_{\infty}\left(\tau_{1}\right) \rightarrow M_{\infty}(\sigma)$ is surjective by exactness of $M_{\infty}(\cdot)$, and so the bottom row is surjective. By assumption, $M_{\infty}\left(\tau_{1}\right) / \mathfrak{m}_{R}$ is one dimensional and $M_{\infty}(\sigma) \neq 0$, and so the bottom row is an isomorphism. We conclude that the composition $M_{\infty}\left(\tau_{2}\right) \rightarrow M_{\infty}\left(\tau_{1}\right) \rightarrow M_{\infty}\left(\tau_{1}\right) / \mathfrak{m}_{R}$ is 0 . 


\section{LATTiCES IN PATChED MODUlES}

In this section, we deduce our main theorem, Theorems 6.1 .3 and 6.2.6, on lattices in cohomology. We again fix a place $v \mid p$ of $F^{+}$and continue to use the notation involving $V_{\widetilde{v}^{\prime}}$ from 5.1 .

6.1. Lattices in patched modules for upper alcove algebraic vectors. Recall the definitions of $W$ and $W^{0}$ from $\$ 2.1, T$ and $T^{\prime}$ from 33.2 , and $R^{\text {alg }}$ from $\$ 5.3$

Lemma 6.1.1. Suppose that $\bar{r}$ satisfies (WE) for $\mu$ at $v$ for some minuscule coweight $\mu$. Then the $R_{\infty}$-module homomorphism $T^{\prime}: M_{\infty}(W) \rightarrow M_{\infty}\left(W^{0}\right)$ induced by $T^{\prime} \in \mathcal{H}\left(W, W^{0}\right)_{\mu}$ is an isomorphism.

Proof. The map $T^{\prime}: M_{\infty}(\bar{W}) \rightarrow M_{\infty}\left(\bar{W}^{0}\right)$ is an isomorphism by Lemma 5.1.5. By Nakayama's lemma, $T^{\prime}: M_{\infty}(W) \rightarrow M_{\infty}\left(W^{0}\right)$ is surjective.

We now prove that $T^{\prime}: M_{\infty}(W) \rightarrow M_{\infty}\left(W^{0}\right)$ is injective. Let $I$ be the kernel of the map $M_{\infty}(W) \rightarrow M_{\infty}\left(W^{0}\right)$. Suppose that $M_{\infty}(\bar{W})$ and $M_{\infty}\left(\bar{W}^{0}\right)$ are nonzero and have support of dimension $d$ as $R_{\infty}$-modules. Let $Z_{d}$ be the functor that assigns to an $R^{\text {alg }}$-module the associated $d$-dimensional cycle (see [EG14, Definition 2.2.5]). We have

$$
Z_{d}\left(M_{\infty}\left(W / p^{n}\right)\right)=Z_{d}\left(M_{\infty}\left(W^{0} / p^{n}\right)\right)
$$

by exactness of $M_{\infty}(\cdot)$ from Theorem 4.1.5 1 , the fact that $\bar{W}$ and $\bar{W}^{0}$ have the same Jordan-Hölder factors, and additivity of $Z_{d}$ in exact sequences.

Let $I_{n}$ be the image of $I / p^{n}$ in $M_{\infty}\left(W / p^{n}\right)$. Then we have the exact sequence

$$
0 \rightarrow I_{n} \rightarrow M_{\infty}\left(W / p^{n}\right) \rightarrow M_{\infty}\left(W^{0} / p^{n}\right) \rightarrow 0
$$

Again by additivity of $Z_{d}$, we see that $Z_{d}\left(I_{n}\right)=0$ and so $I_{n}$ has support of dimension less than $d$. Since $M_{\infty}\left(W / p^{n}\right)$ is maximal Cohen-Macaulay over its scheme-theoretic support, there are no embedded associated primes by [Mat89, Theorem 17.3(i)], and so $I_{n}=0$ and the map $I / p^{n} \rightarrow M_{\infty}\left(W / p^{n}\right)$ must be 0 for all $n$. We conclude that the map $I \rightarrow M_{\infty}(W)$ is 0 and so $I=0$.

Let $V_{v^{\prime}}=W_{v^{\prime}}$ or $W_{v^{\prime}}^{0}$ for $v^{\prime} \neq v$ and $v^{\prime} \mid p$ a place of $F^{+}$. With this fixed choice of $V_{v^{\prime}}$, we continue to use the notation of $\$ 5.1$. By Theorem 5.3.2 we can and do fix an isomorphism $s: R^{\text {alg }} \stackrel{\sim}{\rightarrow} M_{\infty}\left(W^{0}\right)$, which also gives an isomorphism of $R_{\infty^{-}}$ modules $\left(T^{\prime}\right)^{-1} \circ s: R^{\text {alg }} \stackrel{\sim}{\rightarrow} M_{\infty}(W)$ by Lemma 6.1.1. This induces an $R^{\text {alg-action }}$ on $M_{\infty}(W)$. Consider the element

$$
\left(M_{\infty}\left(W^{0}\right) \oplus M_{\infty}(W), s,\left(T^{\prime}\right)^{-1} \circ s\right) \in \mathcal{M}\left(R^{\mathrm{alg}}\right),
$$

with $\mathcal{M}$ the functor defined in 2.3 .2 . Let $\Lambda: \mathbf{Z}_{p}[x, y] /(x y-p) \rightarrow R^{\text {alg }}$ be the corresponding morphism from Proposition 2.3.5.

Theorem 6.1.2. For each place $v^{\prime} \mid p$, let $\mu_{\widetilde{v}^{\prime}}$ be some minuscule coweight. Suppose that $\bar{r}$ satisfies $\left(\mathbf{M}^{\prime}\right),(\mathbf{R T})$, and (WE) for $\mu_{\widetilde{v}^{\prime}}$ at $\widetilde{v}^{\prime}$. Then $\Lambda(y)=s^{-1} \circ T \circ s$. In other words, in the composition $\mathbf{Z}_{p}[x, y] /(x y-p) \rightarrow R^{\text {alg }} \rightarrow \operatorname{End}_{R^{\mathrm{alg}}}\left(M_{\infty}\left(W^{0}\right)\right)$, the image of $y$ is $T$.

Proof. By Proposition 2.3.5, we have that

$$
\Lambda(y)=\left(\left(T^{\prime}\right)^{-1} \circ s\right)^{-1}\left(\begin{array}{ll}
0 & 0 \\
1 & 0
\end{array}\right) s=s^{-1} \circ T^{\prime} \circ i \circ s=s^{-1} \circ T \circ s .
$$


Theorem 6.1.3. Let $F$ be a CM field in which $p$ splits completely. Let $F^{+}$be its totally real subfield and assume that $F / F^{+}$is unramified at all finite places. Let $r: G_{F} \rightarrow \mathrm{GL}_{3}\left(\mathcal{O}_{E}\right)$ be a Galois representation satisfying $(\mathbf{M})$. Assume further that

- $r$ satisfies (RT);

- for all places $v \mid p$ of $F,\left.r\right|_{F_{\widetilde{v}}}$ is a lattice in a crystalline representation with Hodge-Tate weights $\left(c_{\widetilde{v}}+p+1, b_{\widetilde{v}}+1, a_{\widetilde{v}}-p+1\right)$ with $a_{\widetilde{v}}-b_{\widetilde{v}}>6, b_{\widetilde{v}}-c_{\widetilde{v}}>6$, and $a_{\widetilde{v}}-c_{\widetilde{v}}<p-5$;

- for all places $v \mid p$ of $F^{+},\left.r\right|_{F_{\tilde{v}}}$ is irreducible with $\left.\bar{r}\right|_{I_{\tilde{v}}} \cong \psi \oplus \psi^{p} \oplus \psi^{p^{2}}$ where $\psi \cong \omega_{3}^{-\left(a_{\tilde{v}}+1\right)-p\left(b_{\tilde{v}}+1\right)-p^{2}\left(c_{\tilde{v}}+1\right)}$;

- and the reduction $\bar{r}: G_{F} \rightarrow \mathrm{GL}_{3}\left(k_{E}\right)$ satisfies ( $\left.\mathbf{T W}\right)$.

Let $\lambda_{\widetilde{v}}$ be the trace of $\varphi$ acting on $D_{\text {cris }}\left(\left.r\right|_{G_{F_{\widetilde{v}}}}\right)$. Let $V_{\widetilde{v}}, W_{\widetilde{v}}$, and $W_{\widetilde{v}}^{0}$ be as in $\$ 2.1$ and $\pi$ as in $\$ 4.1 .3$. Then the lattice $\otimes_{v} V_{\widetilde{v}} \cap \pi \subset \otimes_{v} V_{\widetilde{v}}$ is isomorphic to $\otimes_{v \mid p}\left(i\left(W_{\widetilde{v}}^{0}\right)+p^{c_{\widetilde{v}}+p-1} \lambda_{\widetilde{v}} W_{\widetilde{v}}\right) \subset \otimes_{v \mid p}\left(W_{\widetilde{v}} \otimes_{\mathbf{z}_{p}} E\right)$.

Proof. Let $\theta: R^{\text {alg }} \rightarrow E$ be the map corresponding to $r$, defined as follows. Recall that in Remark 4.1.4 we fixed a surjection $R_{\infty} \rightarrow R_{\mathcal{S}}^{\square_{S}}$. The composition of this map and the map $R_{\mathcal{S}}^{\square_{S}} \rightarrow E$ defining $r$ gives a map $\theta^{\prime}: R_{\infty} \rightarrow E$. Note that $M_{\infty}(W) / \operatorname{ker}\left(\theta^{\prime}\right)$ is nonzero for some locally algebraic module $W$ by Theorem 4.1.5 3) and axiom (M). Moreover, $W$ must be isomorphic to $\otimes_{v} W_{\widetilde{v}}$ by local-global compatibility at $p$ as in [BLGGT12, Theorem 1.2]. Hence, the $E$-point given by $\theta^{\prime}$ is an $E$-point of the scheme-theoretic support of $M_{\infty}\left(\otimes_{v} W_{\widetilde{v}}\right)$. In other words, the map $\theta^{\prime}$ factors through the faithful quotient of $R_{\infty}$ acting on $M_{\infty}\left(\otimes_{v} W_{\widetilde{v}}\right)$. Let $\mu=\mu_{1}=(0,0,1)$. Again by [BLGGT12, Theorem 1.2], the double coset operator $\iota_{\widetilde{v}}^{-1}\left(\mathrm{GL}_{3}\left(\mathcal{O}_{\widetilde{v}}\right) t_{\mu} \mathrm{GL}_{3}\left(\mathcal{O}_{\widetilde{v}}\right)\right)$ acts by $\lambda_{\widetilde{v}}$. Then $T_{\widetilde{v}}$ acts on $M_{\infty}\left(\otimes_{v} W_{\widetilde{v}}\right) / \operatorname{ker}\left(\theta^{\prime}\right)$ by $p^{c_{\tilde{v}}+p-1} \lambda_{\widetilde{v}} \in \mathcal{O}$. Thus, there is a map $\theta: R^{\text {alg }} \rightarrow E$ extending $\theta^{\prime}$ such that $\theta\left(T_{\widetilde{v}}\right)=p^{c_{\tilde{v}}+p-1} \lambda_{\widetilde{v}}$ by [EGH13, Corollary 4.4.3], [BLGGT12, Theorem 1.2], Theorem 4.1.5 3), and the unramified local Langlands correspondence.

By [BLGG, Theorem A], $\bar{r}$ satisfies (M'). By Example 5.1.3 $\bar{r}$ satisfies (WE) for $\mu_{1}$ at all places $v \mid p$. By Theorem 6.1.2 the composition $\theta \circ \Lambda$ takes $y_{\widetilde{v}}$ to $p^{c_{\tilde{v}}+p-1} \lambda_{\widetilde{v}}$. By Theorem 4.1.5 3) and Remark 4.1.6. $\operatorname{Hom}_{K}\left(\otimes_{v} W_{\widetilde{v}}^{0} \oplus \otimes_{v} W_{\widetilde{v}}, \pi\right)^{d} \cong$ $\left(M_{\infty}\left(\otimes_{v} W_{\widetilde{v}}^{0}\right) \oplus M_{\infty}\left(\otimes_{v} W_{\widetilde{v}}\right)\right) \otimes_{\theta} E$ as $\mathcal{E}$-modules. We conclude that the $\mathcal{E}^{\text {op }}$-module $\operatorname{Hom}_{K}\left(\otimes_{v} W_{\widetilde{v}}^{0} \oplus \otimes_{v} W_{\widetilde{v}}, \pi\right)$ is isomorphic to the $\mathcal{E}^{\mathrm{op}}$-module corresponding to the lattice $\otimes_{v \mid p}\left(i\left(W_{\widetilde{v}}^{0}\right)+p^{c_{\widetilde{v}}+p-1} \lambda_{\widetilde{v}} W_{\widetilde{v}}\right) \subset \otimes_{v \mid p}\left(W_{\widetilde{v}} \otimes_{\mathbf{z}_{p}} E\right)$ for each place $v \mid p$.

Remark 6.1.4. If instead $\left.\bar{r}\right|_{I_{\tilde{v}}} \cong \psi \oplus \psi^{p} \oplus \psi^{p^{2}}$ where $\psi \cong \omega_{3}^{-\left(a_{\tilde{v}}+1\right)-p\left(c_{\tilde{v}}+1\right)-p^{2}\left(b_{\tilde{v}}+1\right)}$, then a similar result holds using the Hecke operator corresponding to $\mu=(0,1,1)$.

Corollary 6.1.5. Keep the assumptions of Theorem 6.1.3. Then $-c_{\widetilde{v}}-p+1<$ $\operatorname{val}\left(\lambda_{\widetilde{v}}\right)<-c_{\widetilde{v}}-p+2$.

Proof. Note that the first inequality follows from EGH13, Corollary 4.4.3 and Proposition 4.5.2]. Let $W$ and $W^{0}$ be $W_{\widetilde{v}}$ and $W_{\widetilde{v}}^{0}$, respectively, and let $V_{\widetilde{v}^{\prime}}$ be $W_{\widetilde{v}^{\prime}}$ in the notation of $\$ 5$. Lemma 5.3.6 shows that $i\left(M_{\infty}\left(W^{0}\right)\right) \subset \mathfrak{m}_{R_{\infty}} M_{\infty}(W)$. Thus $T\left(M_{\infty}(W)\right) \subset \mathfrak{m} M_{\infty}(W)$, and we deduce from the proof of Theorem 6.1.3 that $p^{c \tilde{v}+p-1} \lambda_{\widetilde{v}} \in \varpi \mathcal{O}_{E}$. On the other hand, Proposition 2.1.2, Lemma 5.2.4 and Lemma 5.3.6 show that $p M_{\infty}(W) \subset \mathfrak{m} i\left(M_{\infty}\left(W^{0}\right)\right)$. We deduce that $p M_{\infty}(W) \subset$ $\mathfrak{m} T\left(M_{\infty}(W)\right)$, and that $p^{-c_{\widetilde{v}}-p+2} \lambda_{\widetilde{v}}^{-1} \in \varpi_{E} \mathcal{O}_{E}$. 
6.2. Lattices in patched modules for principal series types. In this section, we prove Theorem 1.1.1 in the case of principal series types. We keep the notation of $\$ 2.2 .2$ and $\$ 5.3 .2$ such as $\tau_{\widetilde{v}^{\prime}}^{s}$ for $s \in S_{3}$ and $\tau_{\widetilde{v}^{\prime}}^{i}$ for $i=1,2,3$. We continue to assume that $\bar{r}$ satisfies (M'), (RT), and (WE) for $\bar{\tau}_{\widetilde{v}^{\prime}}$ and $\widetilde{v}^{\prime}$ for all $v^{\prime} \mid p$.

Fix a place $v \mid p$ of $F^{+}$. Fix $s_{v^{\prime}} \in S_{3}$ for places $v^{\prime} \mid p$ of $F^{+}$with $v^{\prime} \neq v$. From now on we denote $M_{\infty}\left(V_{\widetilde{v}^{\prime}} \otimes \otimes_{v^{\prime} \neq v} \tau_{\widetilde{v}^{\prime}}^{s_{v^{\prime}}}\right)$ by $M_{\infty}(V)$ where $V$ is a $\mathrm{GL}_{3}\left(\mathcal{O}_{\widetilde{v}}\right) \cong \mathrm{GL}_{3}\left(\mathbf{Z}_{p}\right)$ representation.

Proposition 6.2.1. The map $\iota_{r_{j} s_{2}}^{s_{i} s_{2}}: M_{\infty}\left(\tau^{r_{j} s_{2}}\right) \rightarrow M_{\infty}\left(\tau^{s_{i} s_{2}}\right)$ is an isomorphism.

Proof. The map is injective by Theorem 4.1.5 1). By Proposition 2.2.2 and (WE) for $\tau_{v}$, the cokernel of $\iota_{r_{j} s_{2}}^{s_{i} s_{2}}: \bar{\tau}^{r_{j} s_{2}} \hookrightarrow \bar{\tau}^{s_{i} s_{2}}$ contains no Serre weights in $W_{v}(\bar{r})$. By Proposition 5.1.1, the cokernel of $\iota_{r_{j} s_{2}}^{s_{i} s_{2}}: M_{\infty}\left(\bar{\tau}^{r_{j} s_{2}}\right) \rightarrow M_{\infty}\left(\bar{\tau}^{s_{i} s_{2}}\right)$ is 0 . By Nakayama's lemma, the cokernel of $\iota_{r_{j} s_{2}}^{s_{i} s_{2}}: M_{\infty}\left(\tau^{r_{j} s_{2}}\right) \hookrightarrow M_{\infty}\left(\tau^{s_{i} s_{2}}\right)$ is 0 .

The following propositions are proved similarly.

Proposition 6.2.2. The induced map $\iota_{2}^{s_{2}}: M_{\infty}\left(\tau^{2}\right) \hookrightarrow M_{\infty}\left(\tau^{s_{2}}\right)$ is an isomorphism.

For $i=1,2,3$, let $\iota^{i}: \tau^{s_{1}} \oplus \tau^{w_{0}} \rightarrow \tau^{i}$ denote the sum of $\iota_{s_{1}}^{i}$ and $\iota_{w_{0}}^{i}$.

Proposition 6.2.3. For $i=1,3$, the map $\iota^{i}: M_{\infty}\left(\tau^{s_{1}} \oplus \tau^{w_{0}}\right) \rightarrow M_{\infty}\left(\tau^{i}\right)$ is surjective.

Using Theorem 5.3.5, we can and do choose an isomorphism $\psi^{s_{2}}: R^{\mathrm{ps}} \stackrel{\sim}{\rightarrow}$ $M_{\infty}\left(\tau^{s_{2}}\right)$. The compositions $\psi^{r_{i} s_{2}}:=\nu_{s_{2}}^{r_{i} s_{2}} \circ \psi^{s_{2}}: R^{\mathrm{ps}} \stackrel{\sim}{\rightarrow} M_{\infty}\left(\tau^{s_{2}}\right) \stackrel{\sim}{\rightarrow} M_{\infty}\left(\tau^{r_{i} s_{2}}\right)$ are also isomorphisms by Proposition 3.3.1.

For each $i$, Proposition 6.2 .1 gives an isomorphism $\psi^{s_{i} s_{2}}:=\iota_{r_{j} s_{2}}^{s_{i} s_{2}} \circ \psi^{r_{j} s_{2}}: R^{\mathrm{ps}} \stackrel{\sim}{\rightarrow}$ $M_{\infty}\left(\tau^{r_{j} s_{2}}\right) \stackrel{\sim}{\rightarrow} M_{\infty}\left(\tau^{s_{i} s_{2}}\right)$. This also gives an isomorphism

$$
\psi^{w_{0} s_{2}}:=\nu_{s_{1} s_{2}}^{w_{0} s_{2}} \circ \psi^{s_{1} s_{2}}: R^{\mathrm{ps}} \stackrel{\sim}{\rightarrow} M_{\infty}\left(\tau^{w_{0} s_{2}}\right) .
$$

Through these isomorphisms, we endow $M_{\infty}\left(\otimes_{v^{\prime}} \tau_{v^{\prime}}^{s_{v^{\prime}}}\right)$ with the structure of an $R^{\mathrm{ps}}$ module for all choices of $\left(s_{v^{\prime}}\right)_{v^{\prime}}$.

Lemma 6.2.4. We have the following identities:

(1) $\left(\psi^{s_{2}}\right)^{-1} \circ \iota_{r_{i} s_{2}}^{s_{2}} \circ \psi^{r_{i} s_{2}}=\left(\psi^{s_{2}}\right)^{-1} U_{j} \psi^{s_{2}}$;

(2) $\left(\psi^{s_{2}}\right)^{-1} \circ \iota_{s_{i} s_{2}}^{s_{2}} \circ \psi^{s_{i} s_{2}}=\left(\psi^{s_{2}}\right)^{-1} U_{i} \psi^{s_{2}}$; and

(3) $\left(\psi^{s_{2}}\right)^{-1} \circ \iota_{w_{0} s_{2}}^{s_{2}} \circ \psi^{w_{0} s_{2}}=\left(\psi^{s_{2}}\right)^{-1} U_{1} \psi^{s_{2}} \cdot\left(\psi^{s_{1} s_{2}}\right)^{-1} U_{2} \psi^{s_{1} s_{2}}$.

Proof. For (1), we note that

$$
\left(\psi^{s_{2}}\right)^{-1} \circ \iota_{r_{i} s_{2}}^{s_{2}} \circ \psi^{r_{i} s_{2}}=\left(\psi^{s_{2}}\right)^{-1} \circ \iota_{r_{i} s_{2}}^{s_{2}} \circ \nu_{s_{2}}^{r_{i} s_{2}} \circ \psi^{s_{2}}=\left(\psi^{s_{2}}\right)^{-1} U_{j} \psi^{s_{2}}
$$

using Proposition 3.3.2 For (2), Proposition 2.2.4 gives

$$
\left(\psi^{s_{2}}\right)^{-1} \circ \iota_{s_{i} s_{2}}^{s_{2}} \circ \psi^{s_{i} s_{2}}=\left(\psi^{s_{2}}\right)^{-1} \circ \iota_{s_{i} s_{2}}^{s_{2}} \circ \iota_{r_{j} s_{2}}^{s_{i} s_{2}} \circ \psi^{r_{j} s_{2}}=\left(\psi^{s_{2}}\right)^{-1} \circ \iota_{r_{j} s_{2}}^{s_{2}} \circ \psi^{r_{j} s_{2}} \text {, }
$$

which is $\left(\psi^{s_{2}}\right)^{-1} U_{i} \psi^{s_{2}}$ by (1). For (3),

$$
\begin{aligned}
\left(\psi^{s_{2}}\right)^{-1} \circ \iota_{w_{0} s_{2}}^{s_{2}} \circ \psi^{w_{0} s_{2}} & =\left(\psi^{s_{2}}\right)^{-1} \circ \iota_{s_{1} s_{2}}^{s_{2}} \iota_{w_{0} s_{2}}^{s_{1} s_{2}} \circ \psi^{w_{0} s_{2}} \\
& =\left(\psi^{s_{2}}\right)^{-1} \circ \iota_{s_{1} s_{2}}^{s_{2}} \circ \psi^{s_{1} s_{2}}\left(\psi^{s_{1} s_{2}}\right)^{-1} \iota_{w_{0} s_{2}}^{s_{1} s_{2}} \circ \psi^{w_{0} s_{2}} \\
& =\left(\psi^{s_{2}}\right)^{-1} U_{1} \psi^{s_{2}} \cdot\left(\psi^{s_{1} s_{2}}\right)^{-1} U_{2} \psi^{s_{1} s_{2}},
\end{aligned}
$$


where the first equality uses Proposition 2.2.4 and the third equality uses (2) and the fact that $\left(\psi^{s_{1} s_{2}}\right)^{-1} \iota_{w_{0} s_{2}}^{s_{1} s_{2}} \circ \psi^{w_{0} s_{2}}=\left(\psi^{s_{1} s_{2}}\right)^{-1} U_{2} \psi^{s_{1} s_{2}}$ by an argument similar to the one for (1).

Proposition 6.2.5. Let $\alpha_{1}, \alpha_{2}$, and $\alpha_{3}$ be the eigenvalues of $\varphi$ acting on the $\eta^{a_{v}}$, $\eta^{b_{v}}$, and $\eta^{c_{v}}$-eigenspaces, respectively, of $\mathrm{WD}\left(\left.r^{\vee}\right|_{G_{F_{v}}}\right)^{\mathrm{F}-\mathrm{ss}}$. Then $U_{1}$ and $U_{2}$ act on $\operatorname{Hom}_{\mathrm{GL}_{3}\left(\mathcal{O}_{v}\right)}\left(\tau_{v}^{s}, \pi\right)$ by $\alpha_{s(1)}$ and $\alpha_{s(1)} \alpha_{s(2)} / p$, respectively.

Proof. This follows from the first two paragraphs of the proof of HLM16, Theorem $4.5 .2]$.

Theorem 6.2.6. Let $F$ be a CM field in which p splits completely. Let $F^{+}$be its totally real subfield and assume that $F / F^{+}$is unramified at all finite places. Let $r: G_{F} \rightarrow \mathrm{GL}_{3}\left(\mathcal{O}_{E}\right)$ be a Galois representation satisfying $(\mathbf{M})$. Assume further that

- $r$ satisfies (RT);

- for all places $v^{\prime} \mid p$ of $F^{+},\left.r\right|_{F_{\widetilde{v}^{\prime}}}$ is a lattice in a potentially crystalline representation with Hodge-Tate weights $(0,1,2)$ with tame type $\eta^{-a_{\widetilde{v}^{\prime}}} \oplus \eta^{-b_{\widetilde{v}^{\prime}}} \oplus$ $\eta^{-c_{\widetilde{v}^{\prime}}}$ where $a_{\widetilde{v}}-b_{\widetilde{v}}>6, b_{\widetilde{v}}-c_{\widetilde{v}}>6$, and $a_{\widetilde{v}}-c_{\widetilde{v}}<p-5$;

- for all places $w \mid p$ of $F,\left.\bar{r}\right|_{F_{\widetilde{v}}}$ is irreducible with $\left.\bar{r}\right|_{I_{\widetilde{v}}} \cong \psi \oplus \psi^{p} \oplus \psi^{p^{2}}$ where $\psi \cong \omega_{3}^{-\left(a_{\widetilde{v}}+1\right)-p\left(b_{\widetilde{v}}+1\right)-p^{2}\left(c_{\widetilde{v}}+1\right)}$

- and its reduction $\bar{r}: G_{F} \rightarrow \mathrm{GL}_{3}\left(k_{E}\right)$ satisfies ( $\left.\mathbf{T W}\right)$.

Let $\tau_{\widetilde{v}^{\prime}}, \tau_{\widetilde{v}^{\prime}}^{s}$, etc. be as in \$2.2.2 and $\pi$ as in \$4.1.3. Then the lattice $\left(\otimes_{v^{\prime}} \tau_{\widetilde{v}^{\prime}} \otimes_{\mathbf{z}_{p}}\right.$ $E) \cap \pi \subset \otimes_{v^{\prime}} \tau_{\widetilde{v}^{\prime}} \otimes \mathbf{z}_{p} E$ is isomorphic to a tensor product of lattices with factor at $v$ given by

$$
\begin{aligned}
\tau^{s_{2}} & +\left(\alpha_{1} \alpha_{2} / p\right)^{-1} \iota_{e}^{s_{2}}\left(\tau^{e}\right)+\alpha_{2}^{-1} \iota_{r_{1}}^{s_{2}}\left(\tau^{r_{1}}\right) \\
& +\left(\alpha_{1} \alpha_{2} \alpha_{3} / p\right)^{-1} \iota_{r_{2}}^{s_{2}}\left(\tau^{r_{2}}\right)+\left(\alpha^{\prime}\right)^{-1} \iota_{1}\left(\tau^{1}\right)+\left(\alpha^{\prime}\right)^{-1} \iota_{3}\left(\tau^{3}\right),
\end{aligned}
$$

where $\alpha^{\prime}$ is the one of $\alpha_{1} \alpha_{2} / p$ and $\alpha_{2}$ which has smaller valuation.

Proof. Let $\theta: R^{\mathrm{ps}} \rightarrow \mathcal{O}_{E}$ be the map corresponding to $r$, defined as follows. Recall that by Remark 4.1.4 we chose a surjection $R_{\infty} \rightarrow R_{\mathcal{S}}^{\square_{S}}$. The composition of this map and the map $R_{\mathcal{S}}^{\bigsqcup_{S}} \rightarrow \mathcal{O}_{E}$ defining $r$ gives a map $\theta^{\prime}: R_{\infty} \rightarrow \mathcal{O}_{E}$. Let $\tau_{\widetilde{v}}$ be defined as in 5.3 .2 . The map $\theta^{\prime}$ factors through the faithful quotient of $R_{\infty}$ acting on $M_{\infty}\left(\otimes_{v} \tau_{\widetilde{v}}^{s_{2}}\right)$ since $M_{\infty}\left(\otimes_{v} \tau_{\widetilde{v}}^{s_{2}}\right) / \operatorname{ker}\left(\theta^{\prime}\right)$ is nonzero by Theorem 4.1.5 3), axiom (M), local-global compatibility at $p$ as in BLGGT12, Theorem 1.2], and inertial local Langlands as in EGH13, Proposition 2.4.1(ii)]. We extend $\theta^{\prime}$ to $\theta$ using Proposition 6.2.5.

By [EGS15, Lemma 4.1.2], the factor at $v$ of the lattice $\left(\otimes_{v^{\prime} \mid p} \tau_{\widetilde{v}^{\prime}} \otimes \mathbf{z}_{p} E\right) \cap \pi$ takes the form

$$
\begin{aligned}
\tau^{s_{2}} & +\left(\alpha^{r_{1}}\right)^{-1} \iota_{r_{1}}^{s_{2}}\left(\tau^{r_{1}}\right)+\left(\alpha^{r_{2}}\right)^{-1} \iota_{r_{2}}^{s_{2}}\left(\tau^{r_{2}}\right)+\left(\alpha^{s_{1}}\right)^{-1} \iota_{s_{1}}^{s_{2}}\left(\tau^{s_{1}}\right) \\
& +\left(\alpha^{w_{0}}\right)^{-1} \iota_{w_{0}}^{s_{2}}\left(\tau^{s_{2}}\right)+\left(\alpha^{e}\right)^{-1} \iota_{e}^{s_{2}}\left(\tau^{e}\right)+\alpha^{\prime} \iota_{1}\left(\tau^{1}\right)+\alpha^{\prime \prime} \iota_{2}\left(\tau^{2}\right)+\alpha^{\prime \prime \prime} \iota\left(\tau^{3}\right)
\end{aligned}
$$

for some constants $\alpha^{\bullet}$. As in the proof of 6.1.3 $\bar{r}$ satisfies (M') and (WE) for $\tau_{\widetilde{v}}$ and $\widetilde{v}$ at all places $v \mid p$. Thus, we choose isomorphisms $\psi^{\bullet}$ as before. By Proposition 4.1.5 3 , the isomorphisms $\psi^{s}$ induce identifications $\operatorname{Hom}_{\prod_{v^{\prime}} \mathbf{U}_{\left(\mathcal{O}_{v}^{\prime}\right)}\left(\tau^{s} \otimes\right.}$ $\left.\otimes_{v^{\prime} \neq v} \tau_{\widetilde{v}_{v^{\prime}}}^{s^{\prime}}, \pi\right) \stackrel{\sim}{\rightarrow} \mathcal{O}_{E}$ via $\otimes_{\theta} \mathcal{O}_{E}$ and Schikhof duality.

By Lemma 6.2.4, the map

$$
\mathcal{O}_{E} \cong \operatorname{Hom}_{\prod_{v^{\prime}} \mathbf{U}\left(\mathcal{O}_{v}^{\prime}\right)}\left(\tau^{s_{2}} \otimes \otimes_{v^{\prime} \neq v} \tau_{\widetilde{v}^{\prime}}^{s_{v^{\prime}}}, \pi\right) \rightarrow \operatorname{Hom}_{\prod_{v^{\prime}} \mathbf{U}\left(\mathcal{O}_{v}^{\prime}\right)}\left(\tau^{e} \otimes \otimes_{v^{\prime} \neq v} \tau_{\widetilde{v}^{\prime}}^{s_{v^{\prime}}}, \pi\right) \cong \mathcal{O}_{E}
$$


induced by $\iota_{e}^{s_{2}}$ is multiplication by $\alpha_{1} \alpha_{2} / p$; and so $\alpha^{e}=\alpha_{1} \alpha_{2} / p$. The values for $\alpha^{r_{1}}, \alpha^{r_{2}}, \alpha^{s_{1}}$, and $\alpha^{w_{0}}$ are obtained similarly.

We deduce that $\alpha^{\prime \prime}$ can be taken to be 1 by Proposition 6.2.2. By Proposition 6.2 .3 , for $i=1,3$, either the map

$$
\operatorname{Hom}_{\prod_{v^{\prime}} \mathbf{U}\left(\mathcal{O}_{v}^{\prime}\right)}\left(\tau^{i} \otimes \otimes_{v^{\prime} \neq v} \tau_{\widetilde{v}^{\prime}}^{s_{v^{\prime}}}, \pi\right) \rightarrow \operatorname{Hom}_{\prod_{v^{\prime}} \mathbf{U}\left(\mathcal{O}_{v}^{\prime}\right)}\left(\tau^{s_{1}} \otimes \otimes_{v^{\prime} \neq v} \tau_{\widetilde{v}^{\prime}}^{s_{v^{\prime}}}, \pi\right)
$$

or the map

$$
\operatorname{Hom}_{\prod_{v^{\prime}} \mathbf{U}\left(\mathcal{O}_{v}^{\prime}\right)}\left(\tau^{i} \otimes \otimes_{v^{\prime} \neq v} \tau_{\widetilde{v}^{\prime}}^{s_{v^{\prime}}}, \pi\right) \rightarrow \operatorname{Hom}_{\prod_{v^{\prime}} \mathbf{U}\left(\mathcal{O}_{v}^{\prime}\right)}\left(\tau^{w_{0}} \otimes \otimes_{v^{\prime} \neq v} \tau_{\widetilde{v}^{\prime}}^{s_{\widetilde{v}^{\prime}}}, \pi\right)
$$

is an isomorphism. As the maps $\iota_{w_{0}}^{s_{2}}$ and $\iota_{s_{1}}^{s_{2}}$ factor through $\iota^{i}$, we see that $\alpha^{\prime}$ and $\alpha^{\prime \prime \prime}$ can be taken to be one of $\alpha_{2}$ and $\alpha_{1} \alpha_{2} / p$. Finally, $\operatorname{val}\left(\alpha^{\prime}\right), \operatorname{val}\left(\alpha^{\prime \prime \prime}\right) \leq$ $\min \left(\operatorname{val}\left(\alpha_{2}\right), \operatorname{val}\left(\alpha_{1} \alpha_{2} / p\right)\right)$ since the maps in 6.2.1) (resp. (6.2.2p) are given by multiplication by the quotient of $\alpha_{1} \alpha_{2} / p$ (resp. $\alpha_{2}$ ) by $\alpha^{\prime}$ and $\alpha^{\prime \prime \prime}$, which must be elements of $\mathcal{O}_{E}$. We conclude that $\alpha^{\prime}$ and $\alpha^{\prime \prime \prime}$ can be taken to be the one of $\alpha_{2}$ and $\alpha_{1} \alpha_{2} / p$ which has smaller valuation. This yields the desired formula.

Remark 6.2.7. As in Remark 6.1.4 the case $\left.\bar{r}\right|_{I_{\tilde{v}}} \cong \psi \oplus \psi^{p} \oplus \psi^{p^{2}}$ where $\psi \cong$ $\omega_{3}^{-\left(a_{\tilde{v}}+1\right)-p\left(c_{\tilde{v}}+1\right)-p^{2}\left(b_{\tilde{v}}+1\right)}$ is treated similarly.

\section{REFERENCES}

[And87] Henning Haahr Andersen, Extensions of simple modules for finite Chevalley groups, J. Algebra 111 (1987), no. 2, 388-403. MR 916175 (89b:20089)

[BC09] Joël Bellaïche and Gaëtan Chenevier, Families of Galois representations and Selmer groups, Astérisque (2009), no. 324, xii+314. MR 2656025 (2011m:11105)

[BG09] Kevin Buzzard and Toby Gee, Explicit reduction modulo $p$ of certain two-dimensional crystalline representations, Int. Math. Res. Not. IMRN (2009), no. 12, 2303-2317. MR 2511912

[BG13] _ Explicit reduction modulo $p$ of certain 2-dimensional crystalline representations, II, Bull. Lond. Math. Soc. 45 (2013), no. 4, 779-788. MR 3081546

[BG15] Shalini Bhattacharya and Eknath Ghate, Reductions of Galois representations for slopes in (1,2), Doc. Math. 20 (2015), 943-987. MR 3404215

[BGR15] Shalini Bhattacharya, Eknath Ghate, and Sandra Rozensztajn, Reduction of Galois Representations of slope 1, 2015.

[BLGG] Thomas Barnet-Lamb, Toby Gee, and David Geraghty, Serre weights for $U(n)$, to appear in J. Reine Angew. Math.

[BLGGT12] Thomas Barnet-Lamb, Toby Gee, David Geraghty, and Richard Taylor, Local-global compatibility for $l=p$, I, Ann. Fac. Sci. Toulouse Math. (6) 21 (2012), no. 1, 57-92. MR 2954105

[BP12] Christophe Breuil and Vytautas Paškūnas, Towards a modulo $p$ Langlands correspondence for $\mathrm{GL}_{2}$, Mem. Amer. Math. Soc. 216 (2012), no. 1016, vi+114. MR 2931521

[Bre03] Christophe Breuil, Sur quelques représentations modulaires et p-adiques de $\mathrm{GL}_{2}\left(\mathbf{Q}_{p}\right)$. II, J. Inst. Math. Jussieu 2 (2003), no. 1, 23-58. MR 1955206

[Bre10] $\quad$ The emerging p-adic Langlands programme, Proceedings of the International Congress of Mathematicians. Volume II, Hindustan Book Agency, New Delhi, 2010, pp. 203-230. MR 2827792 (2012k:22024)

[Bre14] Sur un problème de compatibilité local-global modulo $p$ pour $\mathrm{GL}_{2}$, J. Reine Angew. Math. 692 (2014), 1-76. MR 3274546

[Bus95] Colin J. Bushnell, Smooth representations of p-adic groups: the role of compact open subgroups, Proceedings of the International Congress of Mathematicians, Vol. 1, 2 (Zürich, 1994), Birkhäuser, Basel, 1995, pp. 770-779. MR 1403977

[CDP14] Pierre Colmez, Gabriel Dospinescu, and Vytautas Paškūnas, The p-adic local Langlands correspondence for $\mathrm{GL}_{2}\left(\mathbb{Q}_{p}\right)$, Camb. J. Math. 2 (2014), no. 1, 1-47. MR 3272011 
$\left[\mathrm{CEG}^{+}{ }^{16}\right]$ Ana Caraiani, Matthew Emerton, Toby Gee, David Geraghty, Vytautas Paškūnas, and Sug Woo Shin, Patching and the p-adic local Langlands correspondence, Camb. J. Math. 4 (2016), no. 2, 197-287. MR 3529394

[CHT08] Laurent Clozel, Michael Harris, and Richard Taylor, Automorphy for some l-adic lifts of automorphic mod $l$ Galois representations, Publ. Math. Inst. Hautes Études Sci. (2008), no. 108, 1-181, With Appendix A, summarizing unpublished work of Russ Mann, and Appendix B by Marie-France Vignéras. MR 2470687 (2010j:11082)

[CL76] R. W. Carter and G. Lusztig, Modular representations of finite groups of Lie type, Proc. London Math. Soc. (3) 32 (1976), no. 2, 347-384. MR 0396731 (53 \#592)

[Col10] Pierre Colmez, Représentations de $\mathrm{GL}_{2}\left(\mathbf{Q}_{p}\right)$ et $(\phi, \Gamma)$-modules, Astérisque (2010), no. 330, 281-509. MR 2642409 (2011j:11224)

[Dia97] Fred Diamond, The Taylor-Wiles construction and multiplicity one, Invent. Math. 128 (1997), no. 2, 379-391. MR 1440309 (98c:11047)

[EG14] Matthew Emerton and Toby Gee, A geometric perspective on the Breuil-Mézard conjecture, J. Inst. Math. Jussieu 13 (2014), no. 1, 183-223. MR 3134019

[EGH13] Matthew Emerton, Toby Gee, and Florian Herzig, Weight cycling and Serre-type conjectures for unitary groups, Duke Math. J. 162 (2013), no. 9, 1649-1722. MR 3079258

[EGS15] Matthew Emerton, Toby Gee, and David Savitt, Lattices in the cohomology of Shimura curves, Invent. Math. 200 (2015), no. 1, 1-96. MR 3323575

[Eme11] Matthew Emerton, Local-global compatibility in the p-adic langlands program for $G L_{2} / \mathbf{Q}$, preprint (2011).

[Fuj06] K. Fujiwara, Deformation rings and Hecke algebras in the totally real case, preprint, 2006.

[Her06] Florian Herzig, The weight in a Serre-type conjecture for tame n-dimensional Galois representations, Ph.D. thesis, Harvard University, 2006.

[Her09] Florian Herzig, The weight in a Serre-type conjecture for tame $n$-dimensional Galois representations, Duke Math. J. 149 (2009), no. 1, 37-116. MR 2541127 (2010f:11083)

[Her11a] , The classification of irreducible admissible mod $p$ representations of a $p$-adic $\mathrm{GL}_{n}$, Invent. Math. 186 (2011), no. 2, 373-434. MR 2845621

[Her11b] A Satake isomorphism in characteristic p, Compos. Math. 147 (2011), no. 1, 263-283. MR 2771132 (2012c:22020)

[HLM16] Florian Herzig, Daniel Le, and Stefano Morra, On mod p local-global compatibility for $\mathrm{GL}_{3}$ in the ordinary case, 2016.

[Jan84] Jens Carsten Jantzen, Filtrierungen der Darstellungen in der Hauptserie endlicher Chevalley-Gruppen, Proc. London Math. Soc. (3) 49 (1984), no. 3, 445-482. MR 759299 (86e:20050)

[Jan03] _ Representations of algebraic groups, second ed., Mathematical Surveys and Monographs, vol. 107, American Mathematical Society, Providence, RI, 2003. MR 2015057 (2004h:20061)

[Kis09] Mark Kisin, Moduli of finite flat group schemes, and modularity, Ann. of Math. (2) 170 (2009), no. 3, 1085-1180. MR 2600871 (2011g:11107)

[Lab11] J.-P. Labesse, Changement de base CM et séries discrètes, On the stabilization of the trace formula, Stab. Trace Formula Shimura Var. Arith. Appl., vol. 1, Int. Press, Somerville, MA, 2011, pp. 429-470. MR 2856380

[Mat89] Hideyuki Matsumura, Commutative ring theory, second ed., Cambridge Studies in Advanced Mathematics, vol. 8, Cambridge University Press, Cambridge, 1989, Translated from the Japanese by M. Reid. MR 1011461 (90i:13001)

[Paš13] Vytautas Paškūnas, The image of Colmez's Montreal functor, Publ. Math. Inst. Hautes Études Sci. 118 (2013), 1-191. MR 3150248

[Tho12] Jack Thorne, On the automorphy of l-adic Galois representations with small residual image, J. Inst. Math. Jussieu 11 (2012), no. 4, 855-920, With an appendix by Robert Guralnick, Florian Herzig, Richard Taylor and Thorne. MR 2979825

[TW95] Richard Taylor and Andrew Wiles, Ring-theoretic properties of certain Hecke algebras, Ann. of Math. (2) 141 (1995), no. 3, 553-572. MR 1333036 (96d:11072)

E-mail address: le@math.toronto.edu

Department of Mathematics, The University of Toronto 